\title{
System EVM Characterization and Coverage Area Estimation of 5G Directive mmW Links
}

\author{
Marko E. Leinonen, Student Member, IEEE, Markku Jokinen, Nuutti Tervo, Student Member, IEEE, \\ Olli Kursu and Aarno Pärssinen, Senior Member, IEEE
}

\begin{abstract}
RF performance of $5 \mathrm{G}$ new radio (NR) millimeter wave $(\mathrm{mmW})$ system will be characterized over-the-air (OTA) in 3GPP 5G NR performance and type approval testing. Total system Error Vector Magnitude (EVM) performance has not been standardized in 3GPP since mobile and base stations are certified separately. In this paper, we extend the OTA measured system EVM concept to cover EVM performance testing with beam steering for $5 \mathrm{G} \mathrm{mmW}$ radio link. We show that the OTA measured system EVM inside of an electro-magnetic compatibility (EMC) chamber can be directly converted to an expected link range, and this has been verified with outdoor measurements. The EVM measurements are performed for a set of beamforming directions to form an expected coverage area. The OTA measured system EVM results confirm that the 5G $\mathrm{mmW}$ Proof-of-Concept radio supports 5G NR 16-QAM and 64-QAM modulations as well as 256-QAM modulation, which is currently specified for sub-6 GHz, only. The maximum estimated range of the $5 \mathrm{G} \mathrm{PoC} \mathrm{mmW} \mathrm{link} \mathrm{is}$ $840 \mathrm{~m}$ with 16-QAM modulation and $150 \mathrm{~m}$ with 256-QAM modulation. The estimated cell coverage with \pm 15 degrees beam steering varies from $205000 \mathrm{~m} 2$ to $6500 \mathrm{~m} 2$ using 16-QAM and 256-QAM modulations, respectively. Usable beam width $(B W)$ of the transceiver array is dependent on the system EVM requirement. It varies based on modulation, coding, and link distance. This phenomenon is called cell breathing, which is similar to any cellular system with adaptive coding techniques, although BW might be much narrower in $5 \mathrm{G} \mathrm{mmW}$ systems.
\end{abstract}

Index Terms - Array EVM, array receiver, OTA noise figure, mmWave, phased array, wireless backhaul.

\section{INTRODUCTION}

$\mathrm{T}$ HE fifth-generation (5G) communication system will offer ten-fold data rates compared with the current long term evolution (LTE) system. The primary enabler for enhanced data rates is that the $5 \mathrm{G}$ system will be deployed at millimeterwave $(\mathrm{mmW})$ bands where wider communication bandwidth can be used. The promise is to provide up to $20 \mathrm{Gbps}$ and 10 Gbps peak data rates for downlink and uplink, respectively. The first trial 5G mmW network was demonstrated in 2018 [1], and the first commercial network was opened in April 2019 [2].

Propagation loss at $\mathrm{mmW}$ frequencies is significantly higher compared to LTE frequencies, and antenna arrays will be used in $5 \mathrm{G} \mathrm{mmW}$ mobile and base stations. Different number of antennas from 32 [3] to 144 [4] or even 384 elements [5] have

This work has been financially supported in part by Academy of Finland 6Genesis Flagship (grant 318927) and in part Business Finland funded 5G Finnish Open Research Collaboration Ecosystem (5G-FORCE). been reported in the literature to support $5 \mathrm{G} \mathrm{mmW}$ operation. Cabling of the antenna array for conducted testing would be very complicated, and the small physical dimensions of the array would make conducted testing unfeasible. For this reason, the $5 \mathrm{G} \mathrm{mmW}$ new radio (NR) standards [6],[7] utilize radiated measurements for characterizing the radio performance.

A system error vector magnitude (EVM) is a combination of transmitter (TX) and receiver (RX) EVMs. In the LTE system, the EVM is measured only at the highest transmission (TX) power level [8]. The RX EVM is used as a design parameter for the RX signal path, and this is validated via bit error rate (BER) or block error rate (BLER) measurements. However, the relationship between BER/BLER and RX EVM is a non-linear function depending on the used signal modulation, coding, digital signal processing, and RX signal level. The EVM gives a more consistent measure of RF performance than BER or BLER. The system EVM performance of the $\mathrm{mmW}$ system at an indoor scenario with multiple beams has been studied in [9].

The system EVM values were measured from the boresight direction of the radio unit without beam steering in our previous work [10]. The measured system EVM can be converted into expected link range, which is one-dimensional metric. This concept can be extended into a two-dimensional measurement to estimate the coverage area of the $5 \mathrm{G} \mathrm{mmW}$ cell by using beam steering. The TX EVM standalone performance has been measured by using 16-QAM single carrier test signal with beam steering at $71 \mathrm{GHz}$ in [11]. 256-QAM modulation has been demonstrated using $80 \mathrm{MHz}$ wide test signal at $28 \mathrm{GHz}$ with conductive measurements in [12].

Currently, transceiver cell coverage testing is performed outdoors with drive testing, and hence it suffers from weather conditions, data traffic of the cell, moving objects like cars, and external interference. Repeatability of such outdoor measurements is poor, and the results are environment dependent. Over-the-air (OTA) testing with a channel emulator inside an electro-magnetic compatibility (EMC) chamber is another possible method, but such systems are not widely available for $\mathrm{mmW}$ frequencies. In $\mathrm{mmW}$ systems, line-of-sight (LOS) propagation conditions are often assumed. In the LOS scenario, the cell coverage estimation can also be done in a laboratory environment with good accuracy due to the relatively static propagation conditions of a directive $\mathrm{mmW}$

Marko E. Leinonen, Markku Jokinen, Nuutti Tervo, Olli Kursu and Aarno Pärssinen are with the University of Oulu, Finland. (e-mail: marko.e.leinonen, markku.jokinen, nuutti.tervo, olli.kursu, aarno.parssinen@oulu.fi).

This paper is an extended version from the IEEE MTT-S International Symposium (IMS2019), Boston, MA, USA, June 2-7, 2019. 
link. Such measurements can be performed inside of the EMC chamber, where the link is isolated from external interference. Laboratory measurements are faster, cheaper, and more repeatable compared to the time-consuming field testing,

The paper is organized as follows. Section II presents an overview of the Proof-of-Concept (PoC) 5G back-haul radio solution. Section III focuses on methods of characterization of the system EVM. Section IV presents the system EVM characterization without beam steering, and Section V presents the system EVM characterization with beam steering using multiple 5G modulations, and corresponding link and coverage range estimations.

\section{IMPLEMENTED 5G MMW RADIO SUPPORTING $28 \mathrm{GHZ}$}

A mobile $5 \mathrm{G} \mathrm{mmW}$ backhaul radio unit has been designed to support frequency band from $26.5-29.5 \mathrm{GHz}$, which is currently called band n257 in 3GPP 5G NR specification [6]. The $5 \mathrm{G} \mathrm{mmW}$ radio unit was publicly demonstrated in the 2018 Winter Olympics in South Korea.

The radio unit has two radio cards, and the block diagram of one radio card is shown in Fig. 1 and a photograph of the radio card in Fig. 2. The radio card is an improved version of the $5 \mathrm{G}$ $\mathrm{mmW}$ radio presented in [13]. The RF architecture is a superheterodyne with an intermediate frequency (IF) at $4 \mathrm{GHz}$. Low-side sub-harmonic mixing is used in both RX and TX. A voltage controlled oscillator ( $\mathrm{VCO}$ ) is running at $6 \mathrm{GHz}$ range, and the local oscillator (LO) frequency is two times the VCO frequency, which is filtered with a high pass filter and fed to a sub-harmonic mixer. The RF solution supports time division duplex (TDD) with RF switches that connect transmission (TX) and reception (RX) signals to a common antenna port located in TX/RX frontend block. RF power distribution is done with a

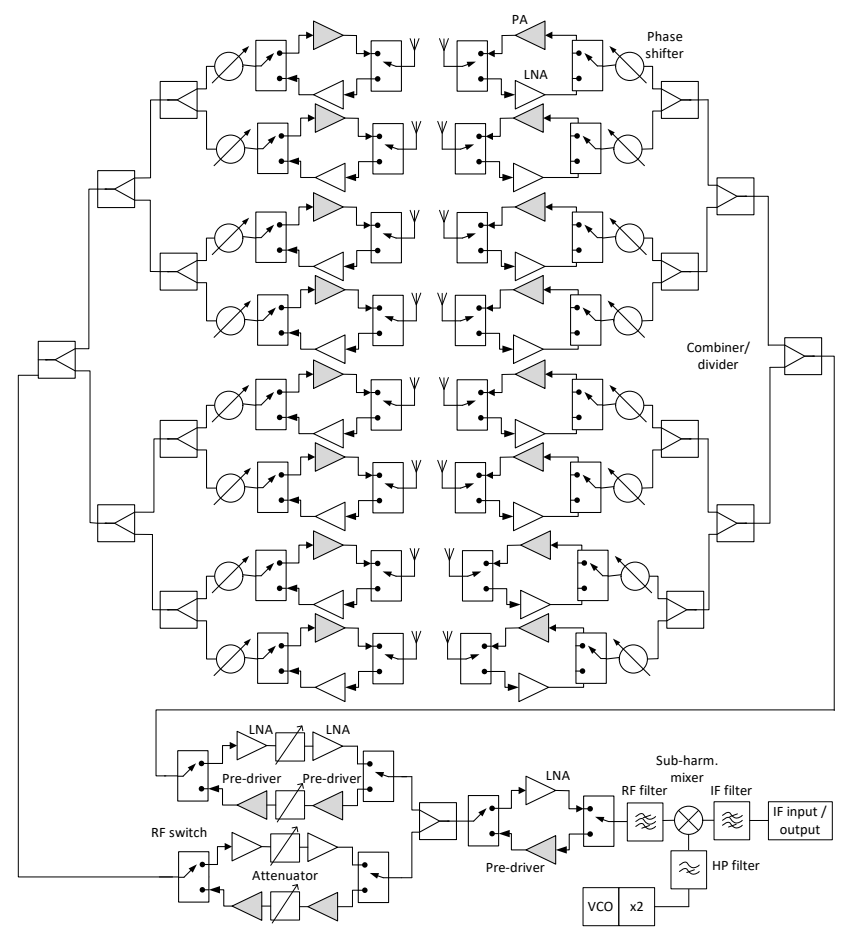

Fig. 1. RF block diagram of the $5 \mathrm{G} \mathrm{mmW}$ backhaul radio unit, where each antenna is a sub-array with $2 \times 2$ elements.

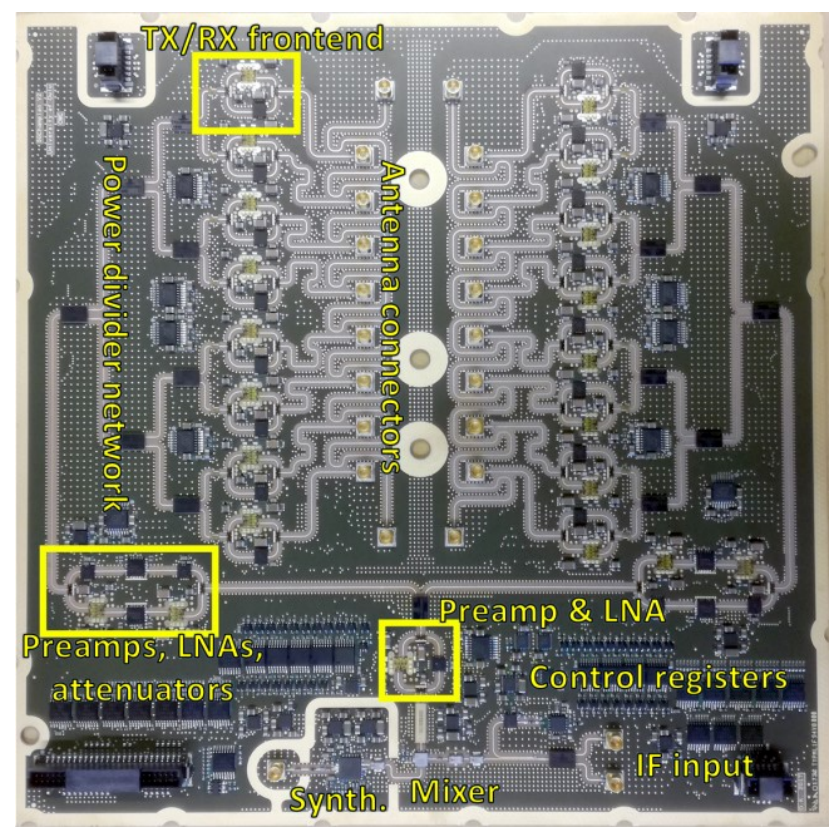

Fig. 2. Photograph of the implemented array transceiver RF card.

tree structure, where power combining is done at $\mathrm{mmW}$ frequency. The radio card includes $16 \mathrm{TX}$ and RX chains, and each TX and RX chain pair has a shared 5-bit digitally controlled phase shifter located at the TX/RX frontend block of Fig. 2. The common path for all chains includes frequency conversion, bandpass filtering of $\mathrm{mmW}$ frequency band, low pass filtering at IF, and power/gain control for TX and RX signals. The radio card has two IF inputs enabling multicarrier combining. The power amplifier used in each TX branch, and the driver stages, is a Gallium Nitride $(\mathrm{GaN})$ based power amplifier (TGA2595), which has a saturated output power $\left(\mathrm{P}_{\text {sat }}\right)$ of $39.5 \mathrm{dBm}$.

Each radio card is connected to an antenna module, including 64 linearly polarized antenna elements shaped in $16 \times 4$ configuration presented in detail in [14]. Hence, each TX and $\mathrm{RX}$ chain is connected to a four-antenna element sub-array via $\mathrm{RF}$ connectors, which are located at the middle of the radio cardin Fig. 2. Antenna element spacing is a half wavelength at

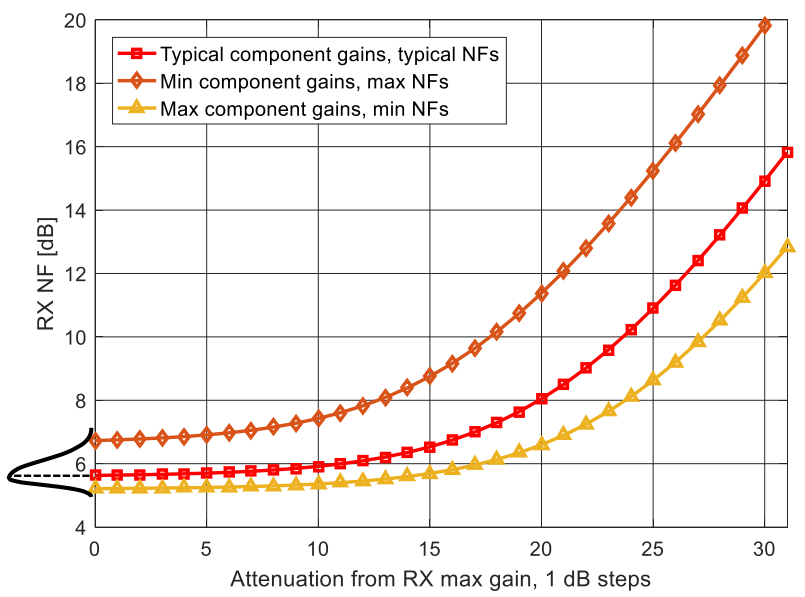

Fig. 3. RF system analysis of NF of array receiver based on component to component variations from data sheets with maximum coherence gain. 

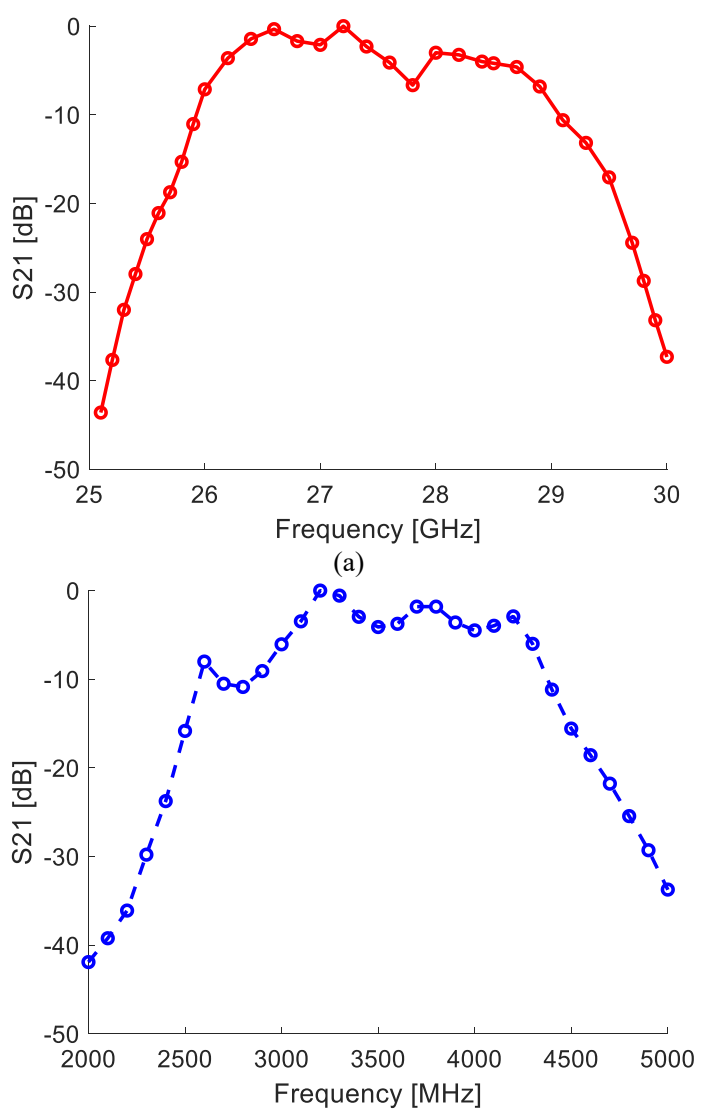

(b)

Fig. 4. Measured filtering performance of the $5 \mathrm{G} \mathrm{mmW}$ radio receiver of (a) $\mathrm{mmW}$ frequency filter and (b) IF frequency filter.

$28 \mathrm{GHz}$ leading to spacing of a wavelength between the fixed four-element subarrays. Due to this RF architecture, the PoC radio unit supports beam steering of \pm 15 degrees without significant sidelobes [15].

An automatic gain control (AGC) can be set for TX and RX separately. The AGC is implemented with digitally controlled 5-bit step attenuators of $31 \mathrm{~dB}$ range at $\mathrm{mmW}$ frequency, as shown in Fig. 1. The AGC functionality is implemented within pre-amplifiers, low noise amplifiers (LNAs), and attenuator block in Fig. 2.

The RF system-level noise figure (NF) of the whole RX array is $5.6 \mathrm{~dB}$, and the NF of one RX path prior to the first power combining is $4.9 \mathrm{~dB}$ with typical component values. Components, including passive ones, after the first power combining, have a minor contribution to the total NF of the RX array. The gain of the RX can be lowered $15 \mathrm{~dB}$ from the maximum RX gain without a significant increase to NF of the whole receiver, as shown in Fig. 3 [10]. Effects of component to component variations to the NF of the array receiver based on component specification limits is shown in Fig. 3. For low gain and high NF values, the NF of the array is $6.7 \mathrm{~dB}$, whereas, with high gain and low NF, the NF of the array is $5.2 \mathrm{~dB}$. A skewed probability density function (PDF) of the array NF with a long tail towards high NF values, as illustrated in Fig. 3.

Filtering of $5 \mathrm{G} \mathrm{mmW}$ radio card has been implemented both at the final $\mathrm{mmW}$ frequency and the IF frequency. A ceramic bandpass $\mathrm{mmW}$ filter with a center frequency of $28 \mathrm{GHz}$ has been used, and the measured frequency response of the receiver is shown in Fig. 4(a). The measurement was done from the antenna connector to the IF connector while varying LO frequency with fixed IF frequency. Low pass and bandpass filters have been used at the IF frequency. The combined measured frequency response of IF filtering is shown in Fig. 4(b). A ripple at the passband of the IF filter can be seen due to non-optimal impedance matching. The measurement was done from the antenna connector to the IF connector with fixed LO frequency while varying IF frequency.

The linearity of the previous version of the receiver array with eight RX chains has been OTA measured by using 16QAM modulated $100 \mathrm{MHz}$ signal in [16]. The RX spectrum regrowth due to RX non-linearity was measured by using adjacent channel power (ACP). The measured and modeled third-order input intercept point (IIP3) was $-4 \mathrm{dBm}$. The IIP3 model third-order non-linearity effect, only, and it omits other non-linearity sources. Two other interference mechanisms in the RX chain were measured from the down-conversion mixer: $\mathrm{LO} / 2-\mathrm{IF}$ and $5 \cdot \mathrm{LO}-2 \cdot \mathrm{RF}$. These mechanisms generate interference products to the RX IF so that the IIP3 is not only a source of non-linearity in the RX.

The radio unit has been a phase calibrated based on OTA measured S-parameters as described in [15]. The control of the antenna beam is implemented with software running on a dedicated processor board within the radio unit. One of the 16 signal paths was selected as a reference path, and phase responses of other paths have been compared to the reference path in both RX and TX modes, separately. The average phase over the $100 \mathrm{MHz}$ operational channel was compared to the reference phase, and the difference has been used as a compensation value in RX and TX modes, since RX and TX modes have dedicated calibration tables. The optimal beam steering values for phase shifters for RX and TX have been calculated for each signal path in the processor unit, and calibration values have been added to the optimal ones to compensate for RF non-idealities.

Automatized OTA S-parameter measurements in RX and TX modes (16 antenna branches and 32 phase shifter values) took together one hour [15]. The calibration measurements were performed separately for RX and TX modes, and the measurements were performed with fixed radiated power levels. Calibration value for phase shifter control word was calculated in Matlab based on the measured S-parameter values. Floating-point numbers have been used in the processor unit to calculate target phase shift values for each TX and RX paths. The target phase values for each RF branch have been calculated by discrete Fourier transform method, which is used to create the beamforming weight-vector to form the radiation pattern [15]. The 5-bit control word has been used from the processor unit to the phase shifter. Calibrations have been performed at room temperature, and ambient operating temperatures have been similar during EMC-laboratory and outdoor measurements.

The radio unit with active radio card consumes $135.0 \mathrm{~W}$ power in $40 \mathrm{dBm}$ TX mode and $30.0 \mathrm{~W}$ power in RX mode. The size of the RF card is $19.0 \times 19.0 \mathrm{~cm}$, and the size of the 
complete RF unit is $37.5 \times 56.0 \times 6.0 \mathrm{~cm}$, including two RF cards and a control board that includes the processor unit, voltage regulation and fan control.

\section{EVM MEASUREMENT METHODS OF THE 5G MMW RADIO}

The distance of a radio link depends on the signal quality over the link, which can be measured with the system EVM. The main contributors for the signal quality are: radiated TX power level, signal attenuation between TX and RX units, NF of the RX, and EVMs of TX and RX. Theory for NF of the antenna array has been presented in [17] and OTA testing of it in [18].

The system EVM is a combination of TX and RX EVMs [9], and the contribution of EVM of the measurement system should be included in the analysis. If EVM contributions are uncorrelated, which is usually a valid assumption, then the EVM contributions can be combined as

$$
\begin{aligned}
& E V M_{\text {system }}\left(P_{R X}\right) \\
& =\sqrt{E V M_{T X}^{2}\left(P_{T X}\right)+E V M_{R X}^{2}\left(P_{R X}\right)+E V M_{R F E q u i p}^{2}}
\end{aligned}
$$

where $E V M_{T X}, E V M_{R X}$, and $E V M_{R F E q u i p}$ are EVMs of TX, RX, and the measurement system, respectively. $P_{\mathrm{TX}}$ is transmission power level, $P_{\mathrm{RX}}$ is received signal level, and EVM is a function of the transmission and reception signal levels. EVM RFEquip is a combined performance of the measurement system (signal generation and analysis) that is measured using signal levels at the input and output of the device under test (DUT). The $E V M_{R F E q u i p}$ can be measured by using reference setups i.e. conductive and OTA, as shown in Figs. 5(a) and 5(b).

The TX power level $P_{T X}$ is defined as effective isotropic radiated power (EIRP) in the mmW OTA measurement

$$
P_{E I R P_{-} T X}=P_{\text {Cond_TX }}+G_{T X_{-} \text {ant }}
$$

where $P_{\text {EIRP }}$ TX is the radiated TX power from the radio unit, $P_{\text {Cond_TX }}$ is a total conducted TX power, and $G_{T X \_a n t}$ is a total TX antenna array gain, including gains of individual antenna elements and the array gain. Two power control schemes were used for the TX power control: power control with variable attenuators with a fixed IF signal level and a fixed attenuation setting with variable IF power level. The $5 \mathrm{G} \mathrm{mmW}$ radio unit was designed to support $60 \mathrm{dBm}$ EIRP power [18], but to guarantee the stability of the TX chain, at least $13 \mathrm{~dB}$ variable attenuation (19 $\mathrm{dB}$ absolute attenuation) was required between the pre-amplifier stages. Due to this practical reason, PA was driven in linear mode with large back-off. The back-off has no impact on the link performance estimation method, but more attention needs to be paid to TX power control if the power stage is driven into non-linear mode. In the experimental setup introduced in the next section, non-linearity is present but mainly dominated by the mixer and PA driver stages. Therefore, the TX power control range of attenuators was also limited to $18 \mathrm{~dB}$ from the available $31 \mathrm{~dB}$. To study non-linear effects, large enough IF power was used in the tests to drive the mixer and the PA driver stages. Also, the IF input power level of the TX was varied $20 \mathrm{~dB}$ from the external signal source having a maximum power of $-2 \mathrm{dBm}$. The maximum achievable stable TX gain in the platform was $14 \mathrm{~dB}$, resulting in $45 \mathrm{dBm}$ EIRP output power of the array.

The radiated TX power has been measured by using a setup shown in Fig. 5(c). The far-field distance of the antenna array [14] is $1.8 \mathrm{~m}$, and the measurement antenna was placed at a $2.5 \mathrm{~m}$ distance from the radio unit. The OTA measurements were performed by using $5 \mathrm{G}$ NR signal at $40 \mathrm{dBm}$ EIRP power. The maximum, stable measured radiated TX power was limited to $45 \mathrm{dBm}$ and still complying with the $-28 \mathrm{~dB}$ adjacent channel power ratio (ACPR) requirement set by the $3 \mathrm{GPP}$ standard. Average conducted TX power level fed into each 16 antenna ports was $14 \mathrm{dBm}$ to achieve $45 \mathrm{dBm}$ EIRP, since the antenna element gain was $9 \mathrm{dBi}$ and the post-PA loss was $2 \mathrm{~dB}$. The average root mean square (RMS) conducted TX power was at a $25.5 \mathrm{~dB}$ back-off from the saturation power of the PA at $45 \mathrm{dBm}$ EIRP. Simulated and measured crest factors for all used 5G NR modulations (16-QAM, 64-QAM, and 256QAM) were $10.9 \mathrm{~dB}$ at $10^{-3}$ peak probability. Modulation order does not have a significant impact on the crest factor due to the dominance of OFDM. Thus, the highest modulation peaks were $15.6 \mathrm{~dB}$ below the $\mathrm{P}_{\text {sat }}$ of the PA at $45 \mathrm{dBm}$ EIRP, which ensures that the TX at the maximum EIRP operates on the linear operational region of PA. Similar TX EVM performance at 40 $\mathrm{dBm}$ and $45 \mathrm{dBm}$ EIPR powers can be expected. However, the maximum transmission level must be lowered in the OTA measurements, since the maximum TX power will compress the $\mathrm{RX}$ and a dynamic range of the EVM measurement is degraded in the laboratory. In the system analysis, received OTA signal

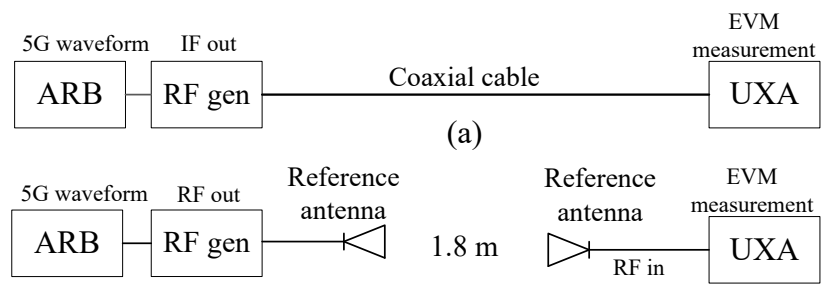

(b)
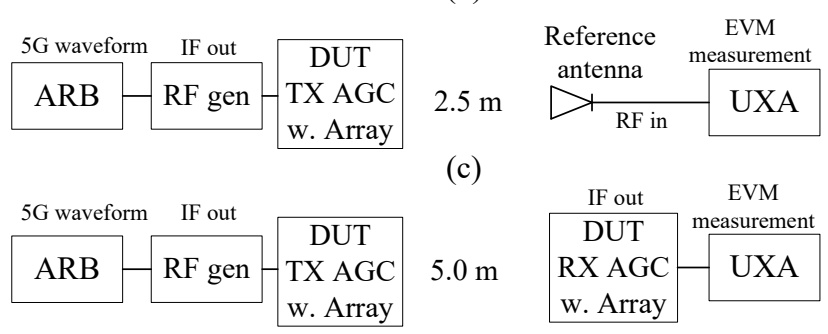

(d)

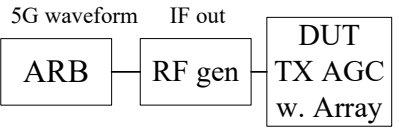

(e)

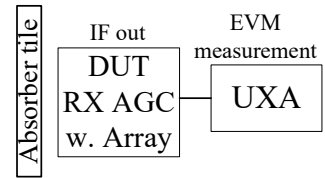

Fig. 5. EVM measurement block diagrams (a) reference conducted EVM (b) reference OTA, (c) TX EVM only, (d) system EVM in EMC chamber and (e) system EVM in EMC chamber with absorber tile [10]. 
level in the receiver can be calculated as

$$
P_{R X_{-} t o t}=P_{E I R P_{-} T X}-F S P L
$$

where $P_{R X_{-} \text {tot }}$ is the received power of receiver of the antenna array, and FSPL is the free space path loss. The received signal level at the output of the array receiver at IF frequency is

$$
P_{R X_{-} \text {out }}=P_{R X_{-} \text {tot }}+G_{R X_{-} \text {ant }}+G_{R X}
$$

where $G_{R R_{-} \text {ant }}$ is the total RX array gain, including gains of individual antenna elements and the array gain, and $G_{R X}$ is a total conducted gain of the array receiver. The conducted gain of the receiver can be varied with attenuator settings of the receiver, and the gain control range of the $\mathrm{RX}$ is $31 \mathrm{~dB}$. The maximum RX gain, including the total RX antenna gain and the conducted gain at maximum setting, is $19.8 \mathrm{~dB}$.

An estimated link distance $D$ can be calculated by extending the free space loss of the measurement distance by attenuating the TX power level and received RX signal level. $D$ can be calculated from the LOS link equation based on received signal level at the output of the receiver as

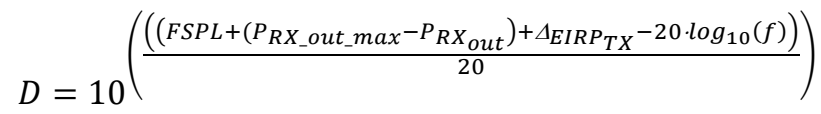

where $P_{R X \text { out max }}$ is the maximum RX output level with the maximum TX power, $P_{R X \_o u t}$ is the measured RX output level at the output of the receiver array, $\Delta_{\text {EIRP }}$ TX is the TX power attenuation from the maximum TX EIRP and $f$ is the operating frequency of $28 \mathrm{GHz}$.

The 5G test signal waveform following the $3 \mathrm{GPP} 5 \mathrm{G}$ NR standard [8] is generated by Keysight M8190A arbitrary waveform generator (ARB), as shown in Fig. 5. The ARB is connected to an RF signal generator (RF gen) Keysight E8267D PSG by differential IQ signal for upconversion to RF or IF. The EVM is measured with Keysight N9040B (UXA) digital signal analyzer and analyzed with Keysight 89600 VSA software. All EVM measurements have been done with $100 \mathrm{MHz}$ wide 16QAM, 64-QAM, or 256-QAM Cyclic-Prefix Orthogonal Frequency Division Multiplexing (CP-OFDM) modulated signals.

The OTA measurements have been done inside a $10 \mathrm{~m} \times 6 \mathrm{~m}$ $\times 5 \mathrm{~m}$ EMC chamber in LOS condition. The size of the EMC chamber fulfills the size requirement for radiated emission testing according to major EMC standards [19]. Measurement inaccuracies have been standardized for $5 \mathrm{G}$ mmW OTA system in [20]. A $95 \%$ confidence interval for TX power measurement is $\pm 1.7 \mathrm{~dB}$, which corresponds to $\pm 2 \sigma$ tolerance area, and thus the allowed measurement uncertainty is $\sigma=0.85 \mathrm{~dB}$ [20].

\section{System EVM MEASUREMENT to BORESIGHT DiRECTION WITHOUT BEAM STEERING}

\section{A. System EVM Measurements in EMC Laboratory}

The system EVM measurement to the boresight of the radio unit without electrical beam steering has been done to validate the proposed system EVM measurement flow, as shown in Fig. 5. First, the conducted reference EVM of the measurement system is verified. The conducted EVM for the system is $0.446 \%$ at $4 \mathrm{GHz}$ IF frequency and $1.776 \%$ at $28 \mathrm{GHz}$ for the measurement setup shown in Fig. 5(a). An additional OTA contribution to the measurement system EVM is measured, as shown in Fig. 5(b) and the contribution is $2.60 \%$ due to, e.g., channel estimation, equalization, and compensation filtering inside of the UXA and potential signal reflections of the EMC lab. Next, the standalone EVM of the TX is measured, as shown in Fig. 5(c). In the standalone TX EVM measurement, the OTA and conducted EVM contributions are subtracted from the TX EVM measurement result. The RX EVM performance can be done, as shown in Fig. 5(d), and the RX EVM can be calculated by subtracting other contributions from the measured system EVM result. Finally, the applicability of AGC based signal level variation is verified with an absorber tile measurement, and the measurement setup is shown in Fig. 5(e).

The system EVM measurements are done by varying the TX and the RX signal levels independently. Measurements were performed with four fixed TX power levels, and the RX signal level was changed within the RX AGC control range at each TX level, as shown in Fig. 6. The power control measurement curves are overlapping in Fig. 6, and the best measured EVM result at RF input level is reported in summary Fig. 7(b). The measured constellation is symmetrical without constellation point rotations, which are typical for phase noise limited radios.

An example of measured EVM constellation with 64-QAM over the OTA link is presented in Fig. 7(a) with the setup shown in Fig. 5(d). A summary of system EVM measurements with 5G NR 64-QAM modulated $100 \mathrm{MHz}$ signal is shown in Fig 7 (b). The measurements have been done in the EMC laboratory at $5 \mathrm{~m}$ distance. The minimum measured total system EVM is $3.90 \%$, when the RX input signal is still on the linear region of the RX input level, as shown in Fig. 7(b). The TX EVM varies from $2.78 \%$ to $4.27 \%$, while the minimum is reached at maximum TX power since the signal to noise ratio of TX is maximized. The measurement system has the smallest contribution $(0.44 \%)$ to the system EVM. The minimum OTA

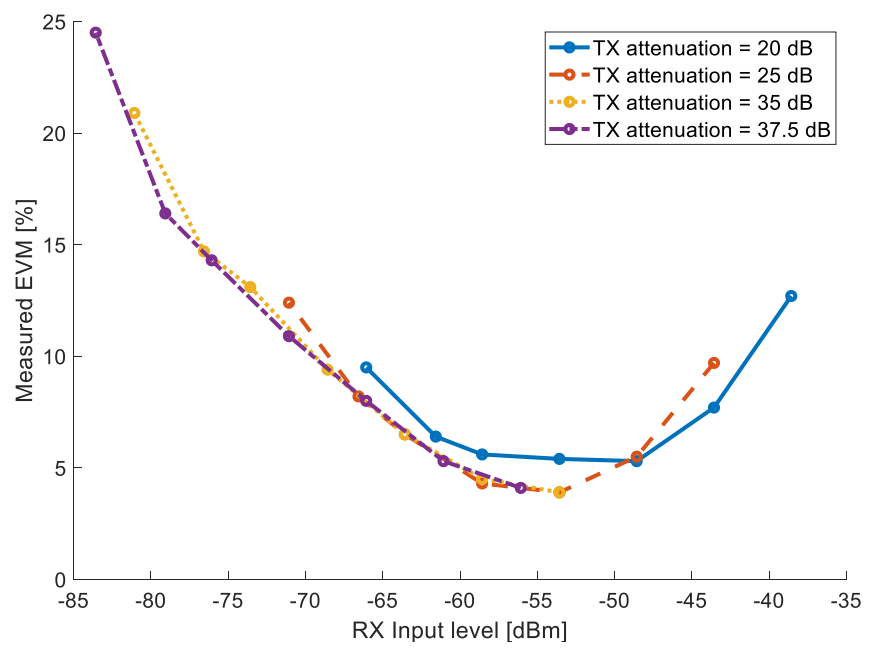

Fig. 6. Used AGC strategy in system EVM OTA measurement with 64-QAM. 
measured RX EVM is $2.34 \%$, which is close to the conducted RX EVM performance (2\%) [13].

The NF of the RX array has been calculated by curve fitting the thermal noise level with varying NF values, as proposed in [18]. The RX NF modeling was done for EVM values between $-58 \mathrm{dBm}$ and $-68 \mathrm{dBm} \mathrm{RX}$ input power levels, where the EVM curve follows a linear 1:1 slope on the logarithm scale. Curve fitting was optimized with the least-squares method for EVMs on a percent scale and a logarithmic scale, and both curve fittings result in a $5.0 \mathrm{~dB}$ NF for the RX array. The measured $\mathrm{RX}$ array $\mathrm{NF}$ of $5.0 \mathrm{~dB}$ follows the calculated maximum component gain and minimum component NF curve in Fig. 3. It can be seen from Fig. 3, that the NF of the RX array increases by $1.5 \mathrm{~dB}$ within the $20 \mathrm{~dB} \mathrm{RX}$ gain control range.

The AGC varied signal level test method was validated by system EVM measurements that were done with maximum TX power with an absorber tile placed between TX and RX units, as shown in Fig. 5(e). Measured attenuation of the absorber tile was $32.8 \mathrm{~dB}$ at $28.0 \mathrm{GHz}$ frequency. The measured system EVM values with the absorber method match with TX and RX AGC varied system EVM measurement results between -70 $\mathrm{dBm}$ and $-81 \mathrm{dBm}$, as shown in Fig. 7(b). Minimum RX attenuation values were used, when the absorber measurement was performed. Thus, the RX and TX gain controls can be used

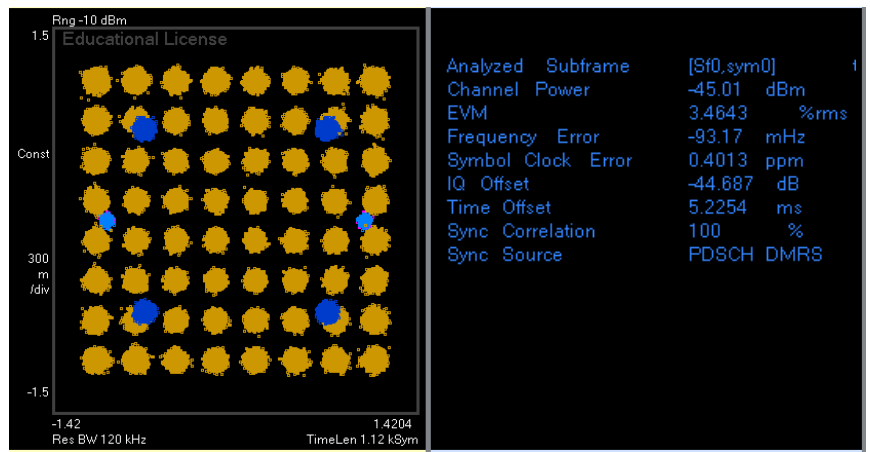

(a)

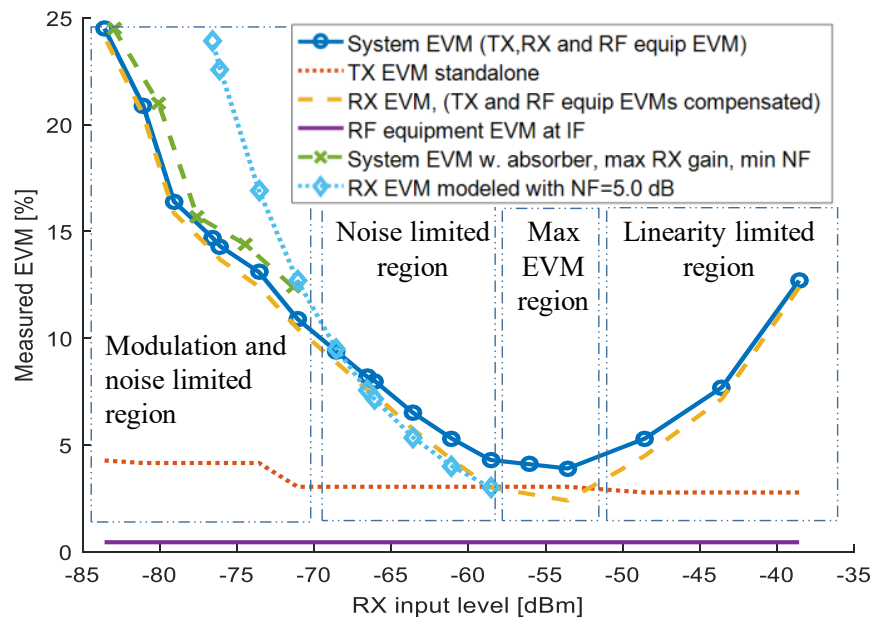

(b)

Fig. 7. System EVM OTA measurement of 5G PoC radio with 5G NR 64-QAM signal at $5 \mathrm{~m}$ distance in EMC laboratory (a) example of measured constellation and (b) summary of system EVM measurements. to control a path loss seen by the signal from the TX to the RX unit or virtually change the physical distance between the RX and TX units. The AGC should be placed in the signal processing chain such that it has only a minor impact to the DUT EVM. Here, a high array gain and the location of the AGC in the common signal path in both at TX and RX signal paths guarantee this condition.

The EVM curve shown in Fig. 7(b) can be divided into four different regions. At the highest RX input signal levels, the linearity of the RX limits the system EVM performance. The system EVM is improved when RX level is decreased, and optimum can be found, when the noise level in the RX is not dominating the performance and received signal is below the compression level. At the noise-limited EVM performance region, the noise level limits the system EVM performance, as shown in Fig. 7(b). The TX EVM performance typically starts to contribute to the system EVM performance at the lowest RX input signal levels when TX is at the highest power.

Additionally, the digital modulation will limit the EVM performance when the RX noise figure performance (light blue curve) deviates from the measured total EVM performance (dark blue curve) in Fig. 7(b). As the order of the modulation increases, the number of ideal modulation points increases, which increases the probability of a random point to be close to any optimal modulation point. Thus, the EVM performance saturates when the order of the digital modulation increases,

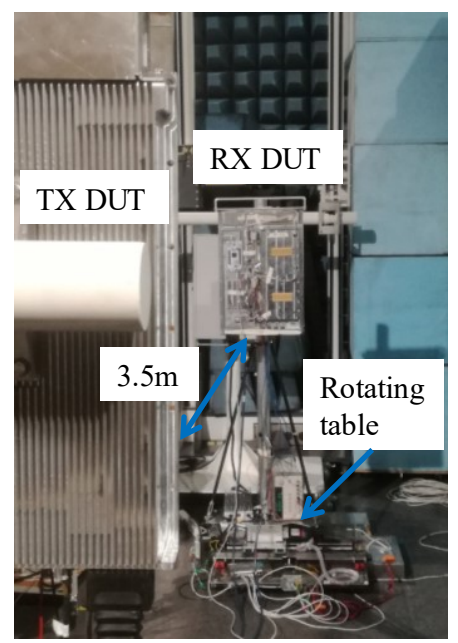

(a)

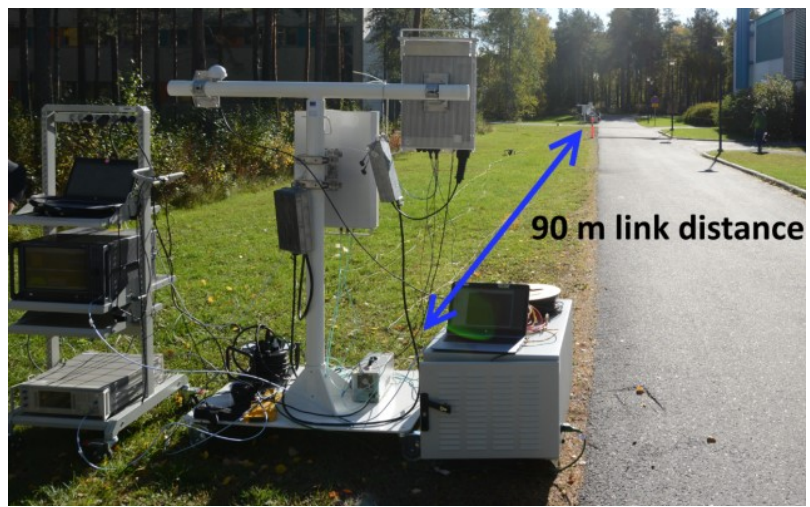

(b)

Fig. 8. 5G mmW PoC testing (a) in EMC chamber and (b) at outdoor. 
which has been demonstrated in [21].

The $10 \mathrm{~dB}$ wide region labeled as max EVM region in Fig 7(b) represents the optimum range of the Rx input signal. The RX EVM (non-idealities of the RX) is dominating the system EVM performance over TX EVM (non-linearity of the TX) in all other the measurement regions than in the optimum EVM range. For this reason, the power control of TX and AGC in the $\mathrm{RX}$ needs to be controlled and optimized carefully during measurements. The RX performance mainly limits the measured system EVM performance, and thus, most of the following performance measurements and analyses are performed from RX point of view.

The BER/BLER provides correct performance metric of the radio link. However, access to such information depends on the modem manufacturer, and it is rarely available. The RF performance is mainly evaluated, for example, in 3GPP standard using a limited set of test cases that are done with white noise channel and predetermined coding. Therefore, in these standardized cases, the relation between RX EVM and BER/BLER is straightforward. The TX performance is specified directly with EVM performance e.g., in 3GPP standard [6].

Statistical measurement system analysis (MSA) is used to validate the repeatability of the measurements and to analyze the impact of the measurement environment to the results. Gage repeatability and reproducibility (Gage R\&R) is a commonly used method to quantify the measurement error [22]. The Gage R\&R method is based on variance analysis, and it can determine variations from the total measured variance introduced by parts, operators, and measurement repeatability, which can be expressed as [22]

$$
\sigma_{T}^{2}=\sigma_{P}^{2}+\sigma_{O}^{2}+\sigma_{R}^{2}
$$

where $\sigma_{T}^{2}$ is the total observed variance of measurement results, $\sigma_{P}^{2}$ is the variance of measured DUTs, $\sigma_{O}^{2}$ is the variance due to operator (or equipment), and $\sigma_{R}^{2} \mathrm{a}$ is the variance due to repeatability. The MSA analysis for the OTA EVM measurement within the EMC chamber has been done with two operators, three sets of 12 repeated measurements and in onehour interval. One DUT has been measured with the same measurement system and with the same system configuration leading the DUT contribution $\sigma_{P}^{2}=0$ in (6). The total observed

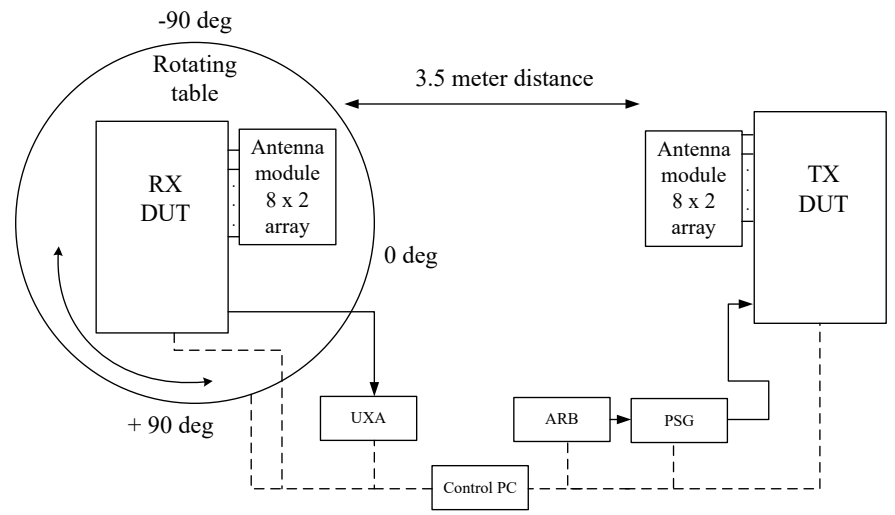

Fig. 9. OTA system EVM measurement setup with beam steering. measurement error in EVM measurement due operators and repeatability is $\sigma_{T}=0.042 \%$. When the measurement error is compared to minimum measured EVM value $3.90 \%$, then the error is $0.042 \% / 3.90 \%=1.07 \%$, which is significantly better than the threshold value $10 \%$ for a good quality measurement system [22].

\section{B. System EVM measurements at Outdoors}

The system EVM measurement has been done outdoors to validate the virtual link EMC chamber measurements. Measurement arrangements for the EMC chamber and outdoor measurements are shown in Fig. 8. The EVM measurement arrangement shown in Fig 8(a) is the same as shown in Fig. 9. The photograph in Fig. 8(a) has been taken from behind of the TX DUT towards the RX DUT direction. The measurement setup for the $90 \mathrm{~m}$ link range experiment is shown in Fig. 8(b).

The EMC chamber measured system EVM values as a function of the expected link range are shown in Fig. 10. The measured system EVM value degradation close to the TX unit can be seen from the measurement results, which is caused by the limited linearity of the RX. The system EVM measurement were done at outdoor at the same TX power level to validate the link range estimations.

Two measurement outdoor measurement campaigns were performed to validate the link ranges estimated in the EMC chamber. The first campaign concentrated on long-range performance up to 90 meters, which was limited by the cable length and signal quality of the synchronization signal. Measurements were performed with 16-QAM and 64-QAM modulations.

The second outdoor measurements concentrated on short distance system EVM performance in order to validate the RX saturation phenomenon with a fixed RX gain. The 64-QAM modulation was used in the measurement, and results are shown in Fig. 10. The system EVM performances of 64-QAM in two different measurements at 30-meter distance are close to each other. A good correlation between the EVM values from the chamber and outdoor measurements can be seen in Fig. 10.

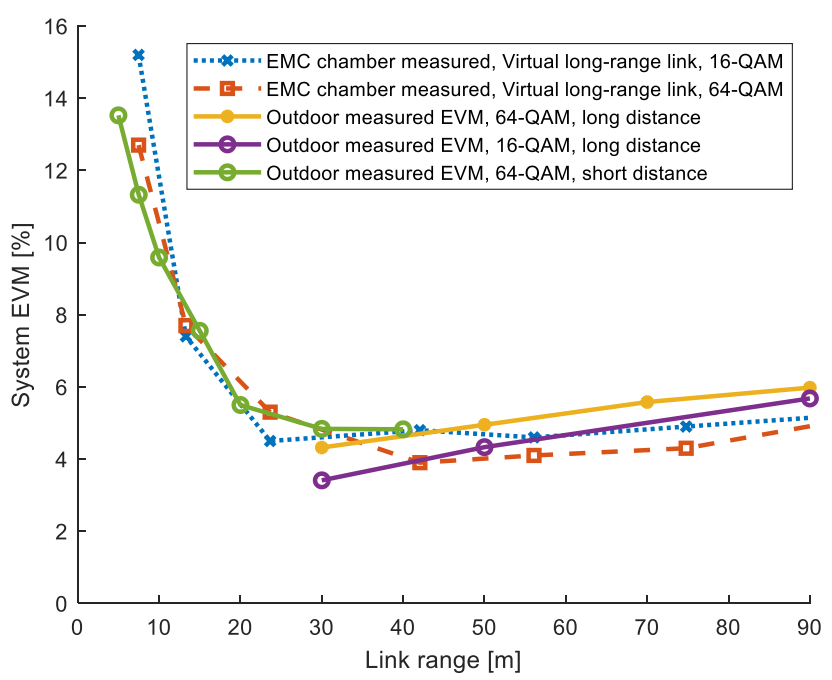

Fig. 10. EMC chamber and outdoor measurement comparison. 
TABLE I

EVM REQUIREMENTS FOR 5G BASE STATION BASED ON 3GPP 38.104

\begin{tabular}{lcc}
\hline \hline Modulation & TX EVM [\%] & Requirement type \\
\hline QPSK & 17.5 & mmW BS, type 2-O, OTA meas. \\
16-QAM & 12.5 & mmW BS, type 2-O, OTA meas. \\
64-QAM & 8.0 & mmW BS, type 2-O, OTA meas. \\
\hline \hline \multirow{2}{*}{ Modulation } & \multirow{2}{*}{ TX EVM [\%] } & Requirement type \\
\hline \hline QPSK & 17.5 & Sub-6 GHz BS, type 1-O, OTA meas. \\
16-QAM & 12.5 & Sub-6 GHz BS, type 1-O, OTA meas. \\
64-QAM & 8.0 & Sub-6 GHz BS, type 1-O, OTA meas. \\
256-QAM & 3.5 & Sub-6 GHz BS, type 1-O, OTA meas. \\
\hline \hline \multirow{2}{*}{ Modulation } & \multirow{2}{*}{ System EVM [\%] } & Notes \\
\hline \hline QPSK & 24.7 & \\
16-QAM & 17.7 & Equal contributions of BS TX EVM \\
64-QAM & 11.3 & and UE RX EVM to system EVM \\
256-QAM & 4.9 & \\
\hline \hline
\end{tabular}

\section{System EVM Measurements and 5G NR Standard}

The TX EVM performance of $5 \mathrm{G}$ base stations (BSs) has been standardized in [6], and a summary is shown in Table I. The TX EVM performance is specified for $5 \mathrm{G} \mathrm{mmW}$ BS up to 64-QAM, but 256-QAM is available for sub-6 GHz BS, only. It should be noted that there is no system EVM requirement in the standard. The system EVM requirement is shown at the bottom of Table I based on modulation properties and Eq. (1) assuming that the system EVM budget is equally shared between the BS TX and the user equipment (UE) RX. This is a valid assumption for TDD backhaul system where both ends of the wireless link are similar, and the maximum EVM performance is ultimately limited by the phase noise performance of the LO. Alternatively, the TX EVM performance can be used as a system EVM specification limit, if the UE is designed to have a much lower RX EVM than the TX EVM of the BS. However, this approach will have a direct impact on the UE component specifications e.g., phase noise level of the RX LO, leading to increasing current consumption compared to balanced EVM contributions case.

The $5 \mathrm{G} \mathrm{mmW} \mathrm{PoC} \mathrm{radio} \mathrm{has} \mathrm{been} \mathrm{designed} \mathrm{to} \mathrm{support}$ signals up to $800 \mathrm{MHz}$ signal bandwidth with eight $100 \mathrm{MHz}$ component carriers (CCs) [13]. The OTA system EVM measurement of eight CCs with 64-QAM was done, and results

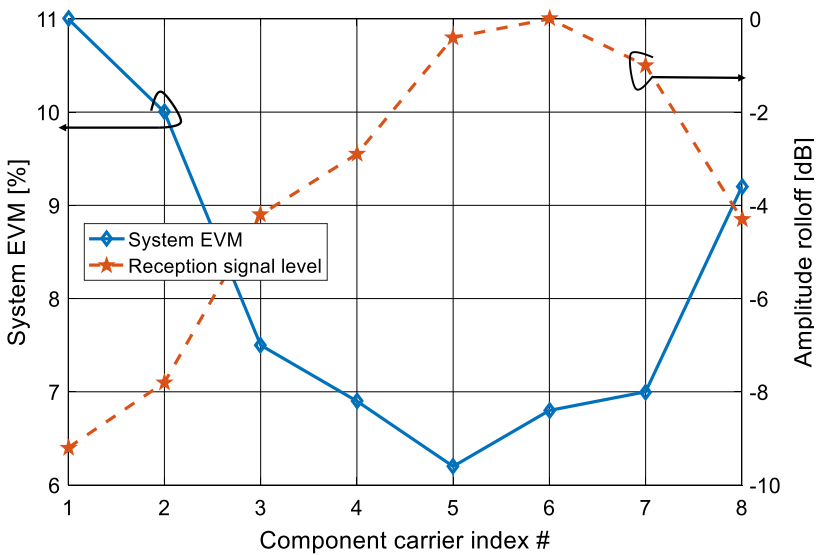

Fig. 11. Measured system EVM of eight 64-QAM $100 \mathrm{MHz}$ component carrier signal on $28.0 \mathrm{GHz}$ center frequency at $-62.6 \mathrm{dBm}$ input level [10]

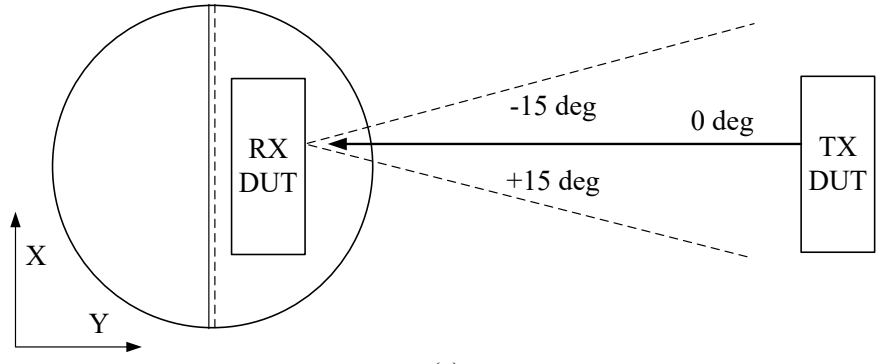

(a)

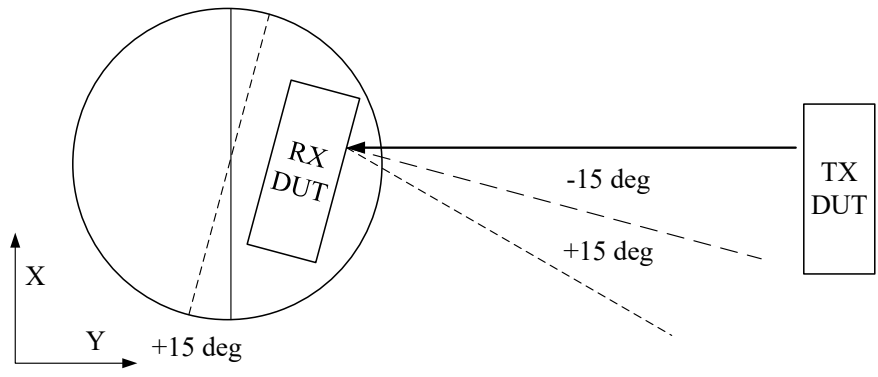

(b)

Fig. 12. Beam steering measurement (a) to the boresight direction and transmission beam is electrically steered \pm 15 degrees and (b) DUT rotated to +15 degree angle and transmission beam is electrically steered \pm 15 degrees.

are shown in Fig. 11. The combined IF frequency responses of the TX and RX create amplitude, and EVM ripples to the measured results over eight $\mathrm{CCs}$. The $5 \mathrm{G}$ PoC radio supports four $100 \mathrm{MHz} 5 \mathrm{G}$ NR carriers (carrier indexes 4 to 7) with similar EVMs and up to 8 carriers with degraded EVM performance. The EVM value is degraded due to amplitude roll-off of combined TX and RX frequency responses, and a combined $-3 \mathrm{~dB}$ system signal bandwidth is $350 \mathrm{MHz}$, as shown in Fig. 11. The four CCs is the maximum configuration in the current 5G NR standard [6].

\section{SySTEM EVM MEASUREMENT WITH BEAM STEERING}

\section{A. Measurement System Setup for OTA Beam Steering}

The $5 \mathrm{G}$ radios will need a beam steering capability to direct narrow transmission and reception beams to optimum directions to enhance communication signal levels and data

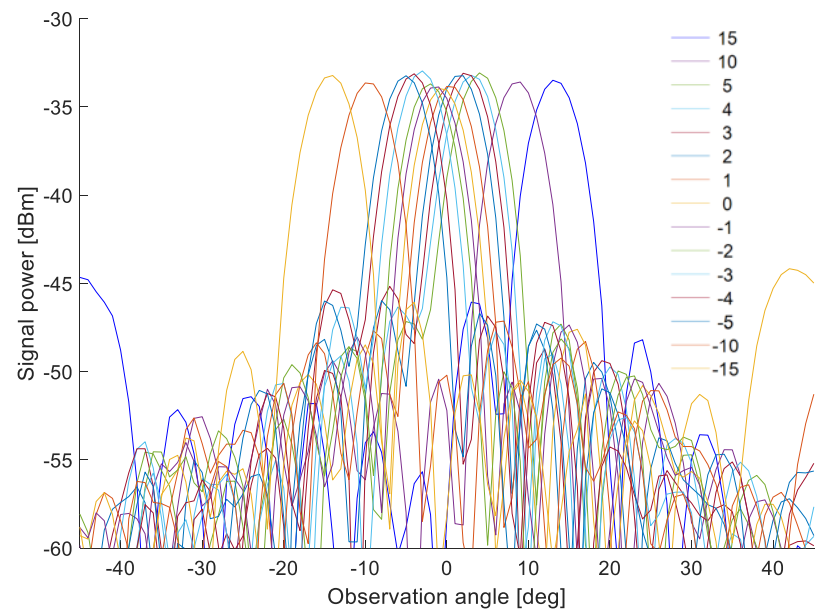

Fig. 13. Measured beam steering performance of the array transceiver with.5G NR 16-QAM modulated $100 \mathrm{MHz}$ wide signal. 
rates. Beam steering performance of the $5 \mathrm{G} \mathrm{mmW}$ radio has been characterized by measurements performed inside the EMC chamber. An overview of the measurement setup is shown in Fig. 9. Two radio units are facing each other spaced $3.5 \mathrm{~m}$ apart. The RX DUT was placed on a rotating table which is controlled by a control PC. The RX DUT can be rotated by 180 degrees, and the location of the TX DUT was fixed during the measurements. The same PC controlled the transmission power of the TX DUT by adjusting the TX IF power level and received power levels of the RX DUT during power level sweeps in the system EVM measurements.

If the RX DUT is rotated without any beam steering capability, the RX beam will be directed away from the TX unit. Thus, the RX beam is electrically steered equally in the opposite direction to compensate the beam misalignment due to the RX DUT rotation, which is illustrated in Fig. 12. The OTA measurement for boresight direction is shown in Fig.12(a), which is the same measurement setup as shown in Fig. 5(d). The $5 \mathrm{G} \mathrm{mmW}$ radio unit \pm 15 degrees beam steering measurements have been performed with 5G NR $100 \mathrm{MHz} 64$ QAM signal, and results are shown in Fig. 13. The grating lobes are located at -45 and +42 degrees, and levels of those are $11.0 \mathrm{dBc}$. Variation of signal power level of the main beam within \pm 15 degrees steering is $0.85 \mathrm{~dB}$. The DUT is capable of $\mathrm{RX}$ and TX electrical beam steering with 1-degree resolution [15],[23]. A steered beam measurement configuration is shown in Fig. 12(b), where RX DUT is rotated +15 degrees in the horizontal direction, and the RX beam is steered \pm 15 degrees. The RX DUT receives the TX signal from the boresight direction when the RX beam is steered -15 degrees at horizontal direction. The DUT positions are fixed in the vertical direction, and there is no RX beam steering in the vertical direction. The OTA system EVM and RX power level measurements have been done with a 5-degrees beam steering step and 1-degree physical rotation step. The effect of increasing NF at high RX attenuation settings has not been compensated in the system EVM analysis.

\section{B. Beamwidth Definition based on EVM measurements}

The most widely used beamwidth definition is the half-power beamwidth (HPBW). The HPBW is the angle between the halfpower $(-3 \mathrm{~dB})$ points of the main lobe, when referenced to the peak effective radiated power of the main lobe [24]. This beamwidth definition assumes that the TX signal covers a

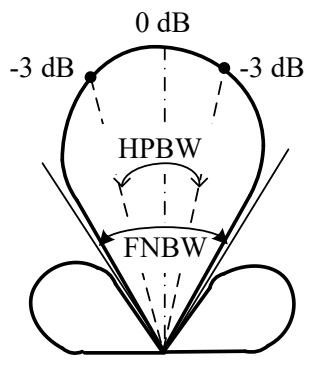

(a)

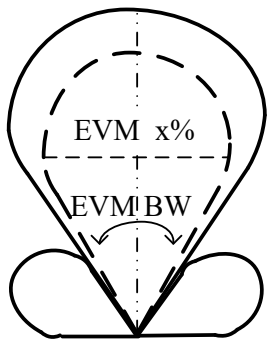

(b)
Fig. 14. Definitions of beamwidth based on (a) half power beamwidth (HPBW) and first null beamwidth (FNBW) and (b) EVM based BW. geographical area, where the signal-to-noise (SNR) ratio can be reduced by $3 \mathrm{~dB}$ from the main lobe direction. However, sometimes, this assumption is not valid due to the used high order digital modulation. The beamwidth definitions are presented in Fig. 14.

Another widely used beam width definition is the first null beamwidth (FNBW), which is the angular width between the first nulls adjacent to the main lobe [24]. The FNBW gives the maximum angular width of the main lobe, but the transmission signal level at the edges of the FNBW is low and cannot support high order digital modulations.

For this reason, a new beamwidth definition is proposed based on the system EVM measurement. The system EVM based beam width is a more realistic cell coverage metric than the HPBW or FNBW, since it is defined based on system performance requirement and not only based on the signal power level.

The system EVM based beamwidth is defined with a contour of measured system EVM value over a coverage area expressed with an observation angle. The system EVM contour is defined as a threshold value with which the measured system EVM needs to comply. The threshold varies based on the used modulation and coding scheme, and thus for one beam with a fixed $-3 \mathrm{~dB}$ BW or HPBW, there may be multiple EVM based beamwidths which is illustrated in Fig. 14(b). The proposed EVM based beamwidth varies based on the received signal quality or RX EVM, which is a function of the link range and received signal power. The EVM based beamwidth changes

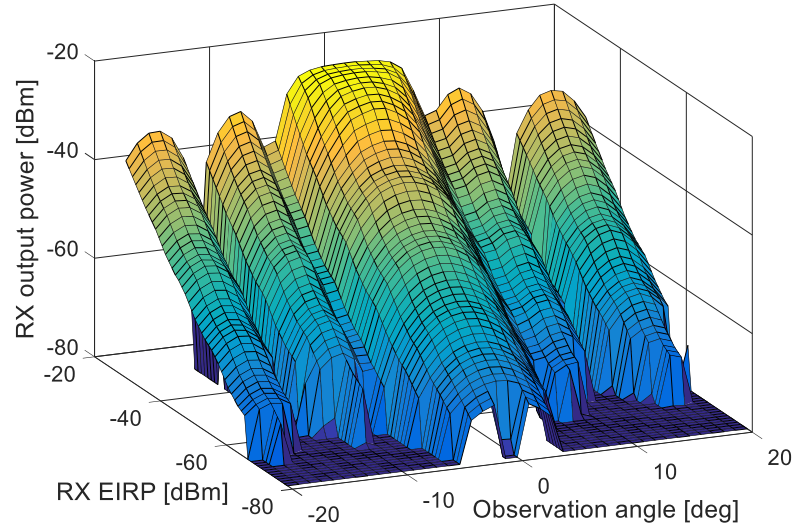

(a)

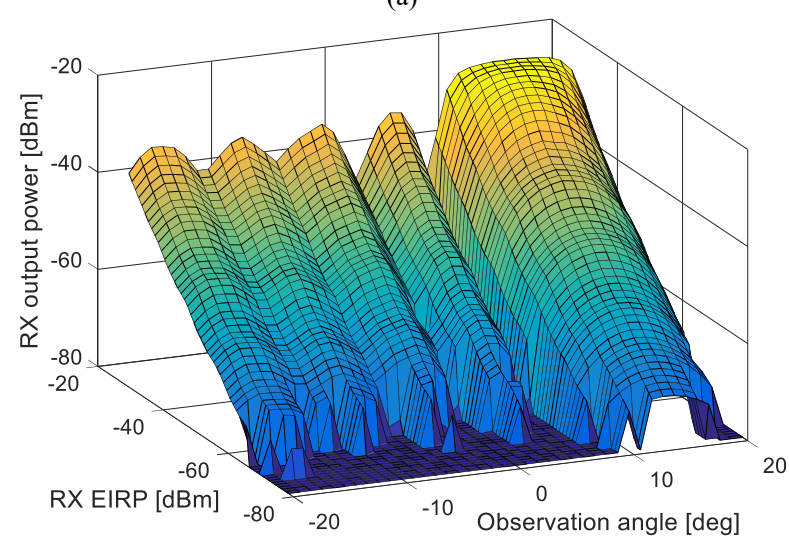

(b)

Fig. 15. Measured RX signal power at the output of the array RX with 5G NR 16-QAM modulated signal with beam steering (a) 0 degree and (b) 15 degrees. 
based on the used modulation, and this is a similar kind of phenomenon as cell breathing in a WCDMA system, where the coverage of the cell changes based on the traffic load of the cell and based on the modulations used by the users [25].

The received signal power measurement results of $5 \mathrm{G}$ NR 16-QAM modulated signal with beam steered to 0 degrees, and 15 degrees are shown in Fig. 15(a) and Fig. 15(b), respectively. The signal level measurement is done at the output of the RX at IF frequency. The HPBW is \pm 3 degrees around the target steering angle, as can be seen from Figs. 15(a) and 15(b). The FNBW is \pm 7 degrees for the signals, as shown in Figs. 15(a) and 15(b), regardless of received signal power level. The same HPBW and FNBW results were measured with 64-QAM and 256-QAM modulations.

The measured system EVM based beamwidths of 16-QAM, 64-QAM, and 256-QAM modulations are compared to HPBW and FNBW in Fig. 16. The HPBW is a reasonable estimate of the beam coverage close to the sensitivity level of the system. The system EVM based signal beam width converges towards FNBW when received signal level increases, and thus the FNBW is a reasonable estimate of beam coverage when estimating coverage at high signal levels. Two system EVM requirements have been studied for EVM beamwidth, and both converge towards FNBW, but shapes of the EVM beams are different. The EVM beam shape at 256-QAM is narrower than the FNBW estimates due to the high SNR requirement to fulfill the EVM threshold value.

\section{System EVM measurement of 5G NR 16-QAM with Beam Steering}

OTA received signal power level measurement at the output of the RX is measured, as shown in Fig. 15. A TX EIRP $40 \mathrm{dBm}$ has been used in the measurements. The received signal power level has been varied with the input signal level of the TX and
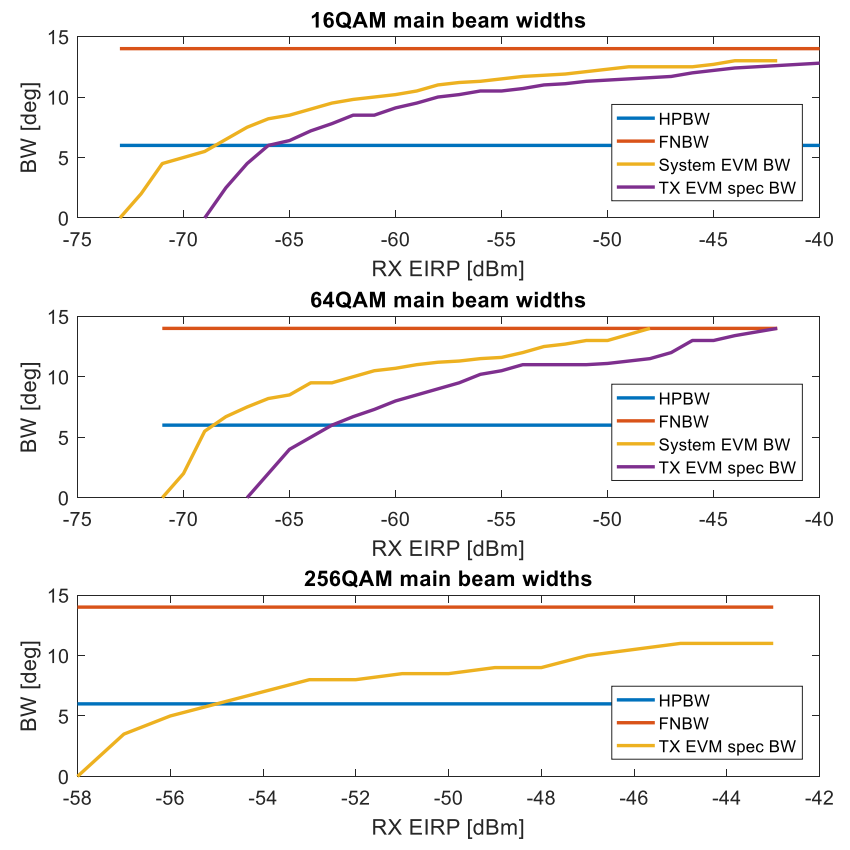

Fig. 16. Measured system EVM based beam widths with different modulations compared to commonly used beamwidth definitions. with attenuators in RX signal paths. The expected received RX signal level at the IF output port of RX has been calculated and compared to measured values at the boresight direction or 0 degree steering angle in Fig. 12(a). A good agreement has been seen over the dynamic range of the measurement. The total standard deviation error between expected and measured results of transmission power is $0.5 \mathrm{~dB}$ over $45 \mathrm{~dB}$ measurement dynamic range, where $20 \mathrm{~dB}$ has been implemented with TX power control and $25 \mathrm{~dB}$ with $\mathrm{RX}$ gain control. The measurement accuracy of the OTA system fulfills the accuracy requirement of the $5 \mathrm{G} \mathrm{NR}$ mmW OTA standard, as described in Section III.

Compression of the received signal at the highest $\mathrm{RX}$ input signal levels can be seen in Fig. 15 as flattening of the main lobe on the six highest RX input signal level values. The sidelobe levels are not compressed as the first sidelobe levels are -14.0 $\mathrm{dBc}$ and $-12.0 \mathrm{dBc}$, and second sidelobe levels are $-15.7 \mathrm{dBc}$ and $-12.3 \mathrm{dBc}$ lower than the main lobe.

The system EVM measurements with \pm 15 degrees beam steering have been performed using a 16-QAM 5G NR signal, and an example EVM measurement result is shown in Fig. 17(a). The electrical beam steering has been done for the RX

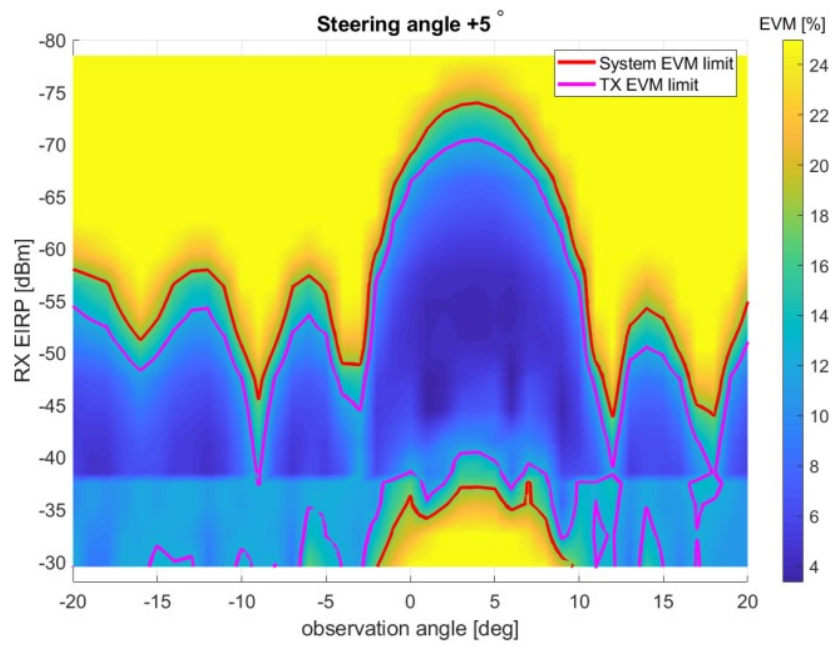

(a)

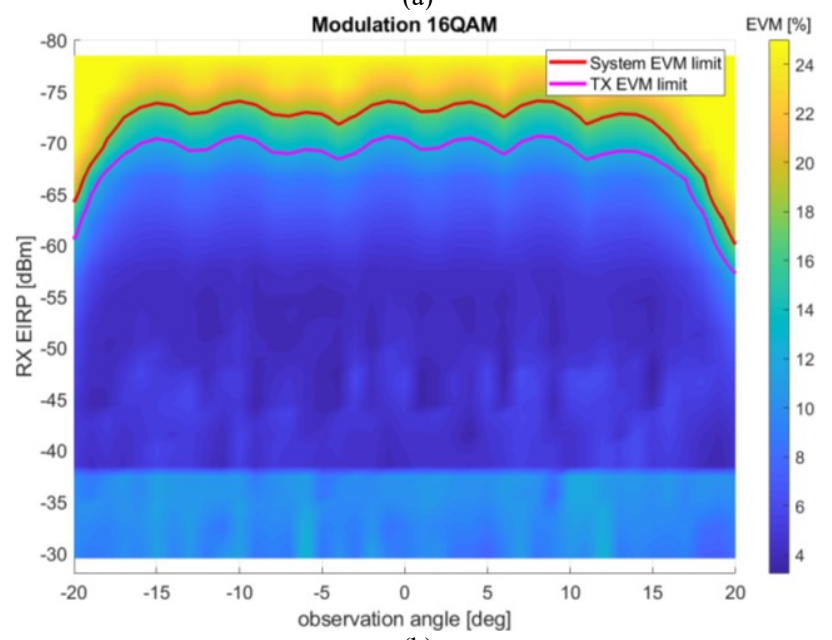

(b)

Fig. 17. 16-QAM system EVM measurement (a) single beam result with 5 degrees steering and (b) combined system EVM result over \pm 15 degrees. 
DUT from -15 degrees to +15 degrees with 5 degrees steps with 1-degree physical rotation, as shown in Fig. 12.

Threshold values for system EVM results are presented in Fig. 17(a) and 17(b) according to Table I. The system EVM limit in the legends on Figs. 17 to 19 and in Figs. 21 and 22 marks the EVM limit where TX EVM and RX EVM have equal contribution to the system EVM. The TX EVM limit in the legends of Figs. 17 to 19 and in Figs. 21 and 22 marks the EVM limit where only the TX EVM is considered and no RX EVM contribution or a perfect RX is assumed.

The shape of the system EVM curve of 16-QAM follows the form of the received signal power, as in Fig. 15, when the threshold value is high. The EVM values are limited to $25 \%$, since beyond this EVM value measurement equipment loses synchronization.

The system EVM performance is impaired at the direction of the main beam at the highest $\mathrm{RX}$ input powers due to compression of the RX or when the RX DUT is nearby to the TX DUT. The shape of the EVM curve at high RX input power levels is not similar to low RX input power levels since the performance of the EVM at high input power levels is affected by non-linear effects [26]. The effect is visible at sidelobes, where the TX EVM threshold limit does not follow the shape

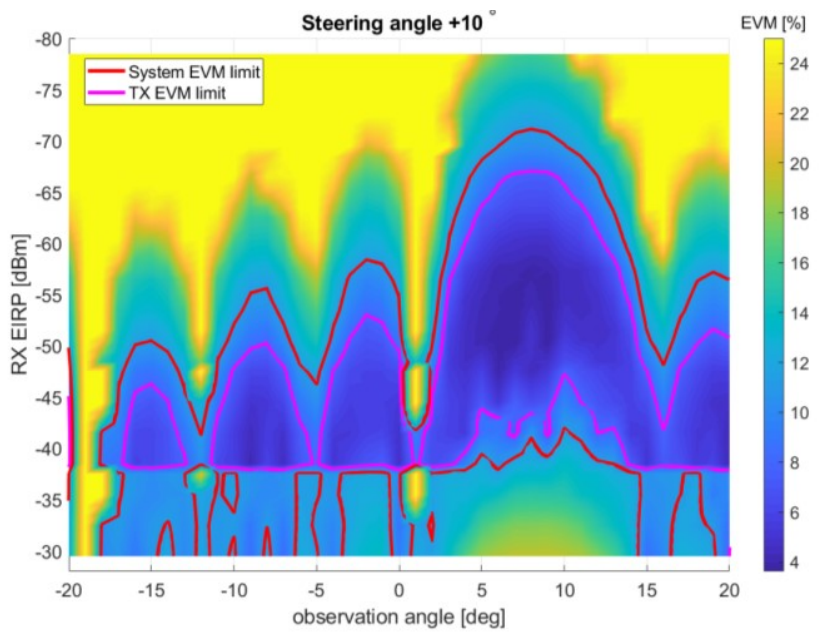

(a)

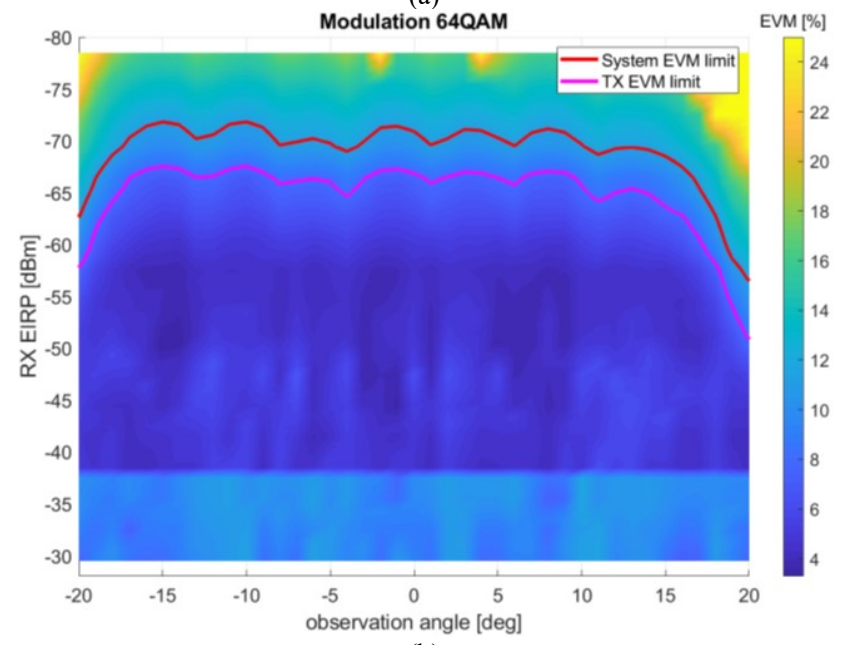

(b)

Fig. 18. 64-QAM system EVM measurement (a) single beam result with 10 degrees steering and (b) combined EVM results over \pm 15 degrees. of the power level. The optimum EVM performance of $3.24 \%$ is achieved with $-55 \mathrm{dBm}$ main beam signal level. The side lobes generate reasonably good coverage around the main beam, which could be used to overcome the problem of RX blocking. One method to do gain control could be that at high input power levels, the RX unit may steer the main beam away from the TX direction and use a side lobe for reception.

A summary of the best EVM values over the beam steering angles is shown in Fig. 17(b). It can be seen from the summary that system EVM values are almost identical over different beam steering angles with 16-QAM modulation.

\section{System EVM Measurement with 5G NR 64-QAM with Beam Steering}

The system EVM measurements with \pm 15 degrees beam steering have been measured with 64-QAM 5G NR signal, and results are shown in Fig. 18. The received RX signal levels at 64-QAM modulation are similar to 16-QAM modulation, which are as shown in Fig. 15.

The shape of the EVM curve of 64-QAM modulation in Fig. 18(a) deteriorates slower than the EVM curve of 16-QAM in Fig. 17(a). Even if the EVM curve of 64-QAM is flatter than 16-QAM, the specification limit for system EVM tightens when the order of the modulation increases to guarantee successful communication, as shown in Table I. The minimum measured EVM value for 64-QAM was $3.29 \%$ in Fig. 18(b). The shape of the EVM curve at low RX input levels follows the form of the power level. Non-linearity effects can be seen at RX input levels above $-45 \mathrm{dBm}$ in the main beam since the EVM response varies in different directions.

A summary of the best EVM values for 64-QAM over the beam steering angles is shown in Fig. 18(b). Measurement steps of the electrical beam steering are visible in Fig. 18(b). The combined threshold value responses of 16-QAM and 64-QAM look similar, even though the curvatures of the EVM responses are different in Figs 17(a) and 18(a).

\section{E. System EVM Measurement with 5G NR 256-QAM with Beam Steering}

The received 256-QAM RX power level curves are very similar to 16-QAM shown in Fig. 15(a), since the same RF settings have been used for all modulations. The system EVM measurements with \pm 15 degrees beam steering have been measured with 256-QAM 5G NR signal. The EVM curve of 256-QAM in Fig. 19(a) is flatter than 64-QAM in Fig. 18(a), as expected. The same standardized EVM performance for sub-6 $\mathrm{GHz}$ frequencies is hard to achieve at $\mathrm{mmW}$ frequencies. The smallest 256-QAM 5G NR OTA measured system EVM value was $3.99 \%$. This measured value fulfills the system EVM specification of $4.5 \%$ from Table I, when equal contribution from TX and RX EVMs is assumed. The measurement results in Fig. 19(a) and 19(b) confirm that 256-QAM could be used at $\mathrm{mmW}$ frequencies with a limited link range.

The needed 256-QAM system EVM performance can be achieved only at the main beam direction, as shown in Fig. 19(a). The EVM curve shape is not following the received signal level, since only three EVM lobes are visible in Fig. 19(a) 
instead of five lobes. The RX blocking performance limits the available signal range more with 256-QAM than with other modulations.

The combined performance of $256-\mathrm{QAM}$ over \pm 15 degrees beam steering is shown in Fig. 19(b). It can be seen from Fig. 19(b) that the measured coverage area is a not uniform. The available signal range for 256-QAM link is limited by the phase noise performance of LOs, TX EVM performance, and nonlinearity of the RX.

\section{F. Cell Coverage with Automatic Beam Steering}

Combined RX signal power levels from each measured beam steering angle with 16-QAM is shown in Fig. 20. The received $\mathrm{RX}$ signal power is substantially constant over the whole beam steering range and the dynamic range of OTA measurements with 16-QAM. The 5-degree electrical steering steps are visible from the results, but the combined received signal $-3 \mathrm{~dB}$ beamwidth is \pm 3 degrees, which covers almost fully the granularity of the electrical step size. Similar combined received RX signal levels are measured with 64-QAM and 256-QAM, since the same RF attenuator and phase shifter settings have been used for all modulations.

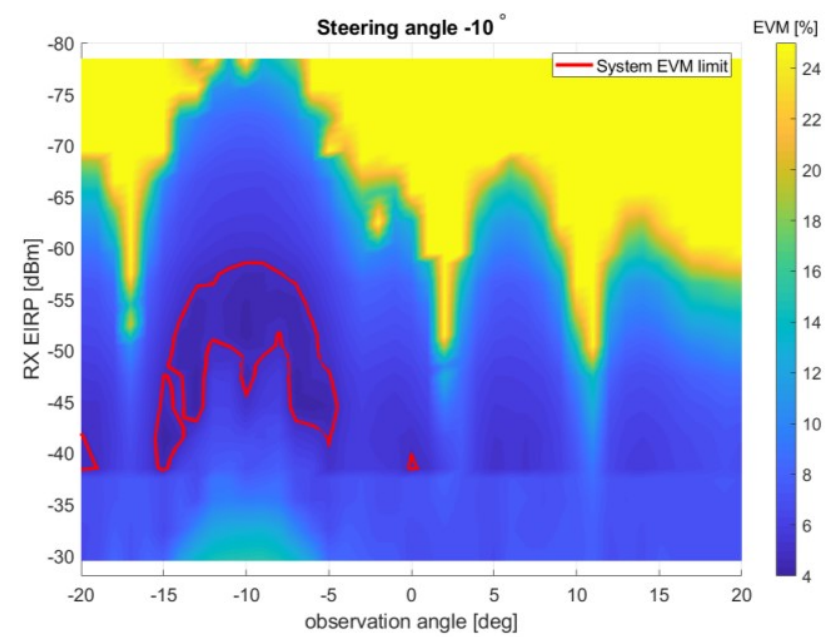

(a)

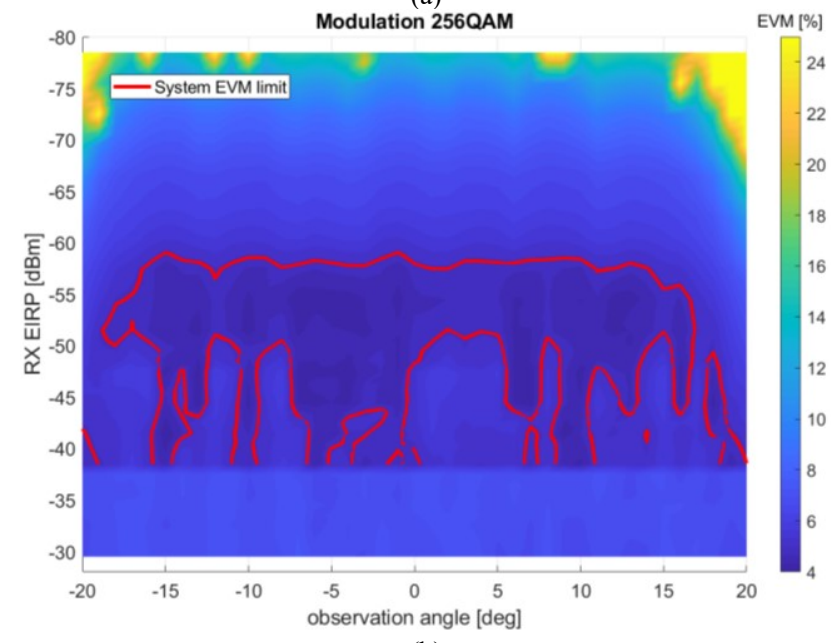

(b)

Fig. 19. 256-QAM system EVM measurement (a) single beam results with -10 degrees steering and (b) combined EVM result over \pm 15 degrees.
A link range estimation for 16-QAM over the beam steering has been done based on measured system EVM performance presented in Fig. 17(b) and applying Eq. (5) to convert the received signal level to the corresponding distance. The link range estimation is shown in Fig. 21(a). Similarly, link ranges are estimated for 64-QAM, which is shown in Fig. 21(b) and for 256-QAM, which is shown in Fig. 21(c). The expected link ranges with $40 \mathrm{dBm}$ TX power using 16-QAM, 64-QAM, and 256-QAM are $475 \mathrm{~m}, 366 \mathrm{~m}$, and $88 \mathrm{~m}$, respectively, with the assumption that the equal contribution system EVM limit from Table I is used.

The expected cell ranges are shorter, if the system EVM threshold is the TX EVM specification with a perfect RX. The cell ranges are $319 \mathrm{~m}$ with 16-QAM and $221 \mathrm{~m}$ with 64-QAM. 256-QAM cannot be supported if the system EVM is limited to TX EVM specification only.

Base stations are classified to different sector classes by how many beams are needed to cover a 360 degrees circle. The implemented \pm 15 degrees horizontal steering corresponds to a 12-sector antenna. Estimated link ranges of 16-QAM, 64-QAM, and 256-QAM presented in Fig. 21 are shown as physical coverage areas in Fig. 22. The coverage areas, with the equal TX and RX contributions to system EVM specification, are $64800 \mathrm{~m}^{2}$ with 16-QAM, $38950 \mathrm{~m}^{2}$ with 64-QAM, and $2200 \mathrm{~m}^{2}$ with 256-QAM. A smoother coverage area prediction would have been reached if electrical beam steering granularity and measurement time had been increased considerably. A complete EVM performance measurement over steering angles took tens of hours to perform with the used granularity.

The ranges and coverages can be improved when the radio unit is tuned to the maximum $45 \mathrm{dBm}$ TX power without TX EVM degradation compared to $40 \mathrm{dBm}$ operation. The cell ranges can be extended up to $840 \mathrm{~m}$ with 16-QAM, $650 \mathrm{~m}$ with 64-QAM, and $150 \mathrm{~m}$ with 256-QAM. Estimated coverage areas for 16-QAM, 64-QAM, and 256-QAM are $205000 \mathrm{~m}^{2}$, $122800 \mathrm{~m}^{2}$, and $6500 \mathrm{~m}^{2}$, respectively.

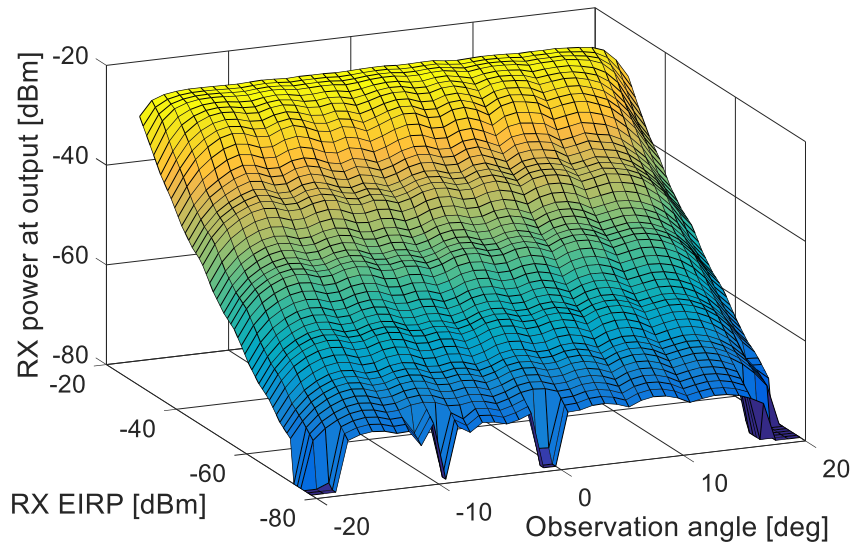

Fig. 20. Combined measured RX signal output power levels of 5G NR 16-QAM with $40 \mathrm{dBm}$ TX EIRP with 5-degree electrical beam steering steps. 


\section{CONCLUSION}

The range of the wireless link is specified by system EVM which is a combination of TX and RX EVMs. Maximum allowed system EVM depends on the used modulation, coding, signal processing algorithms and EVM system partitioning

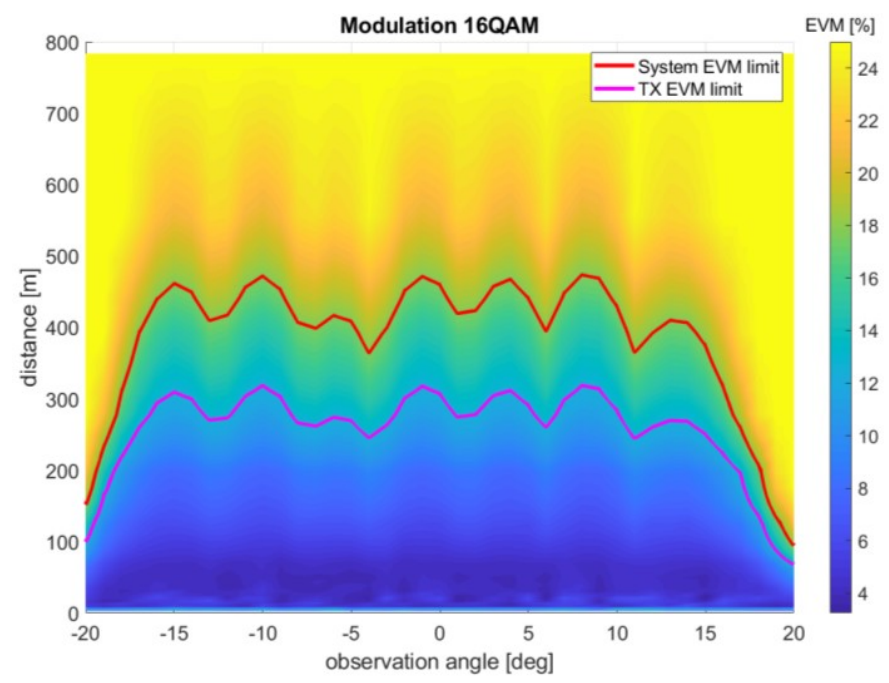

(a)

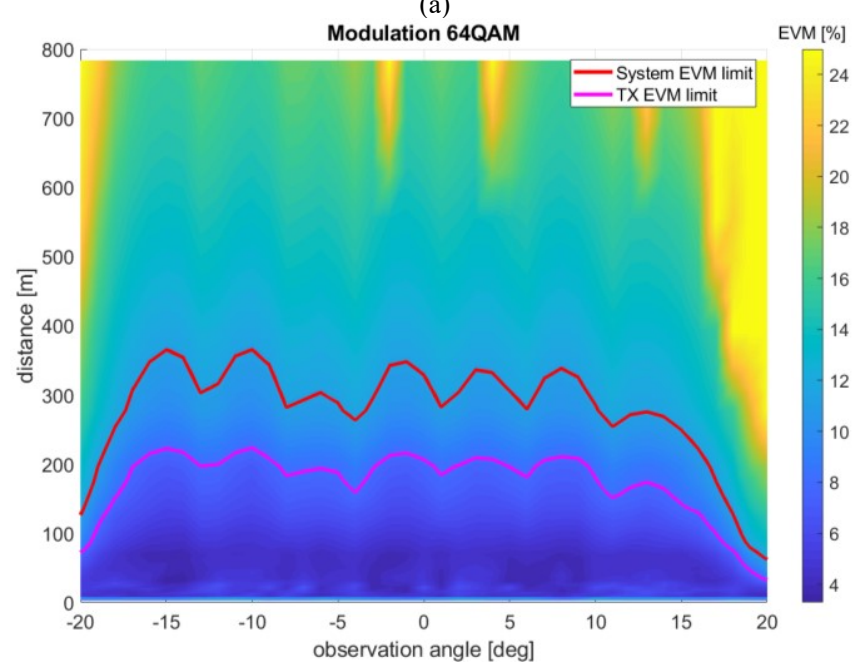

(b)

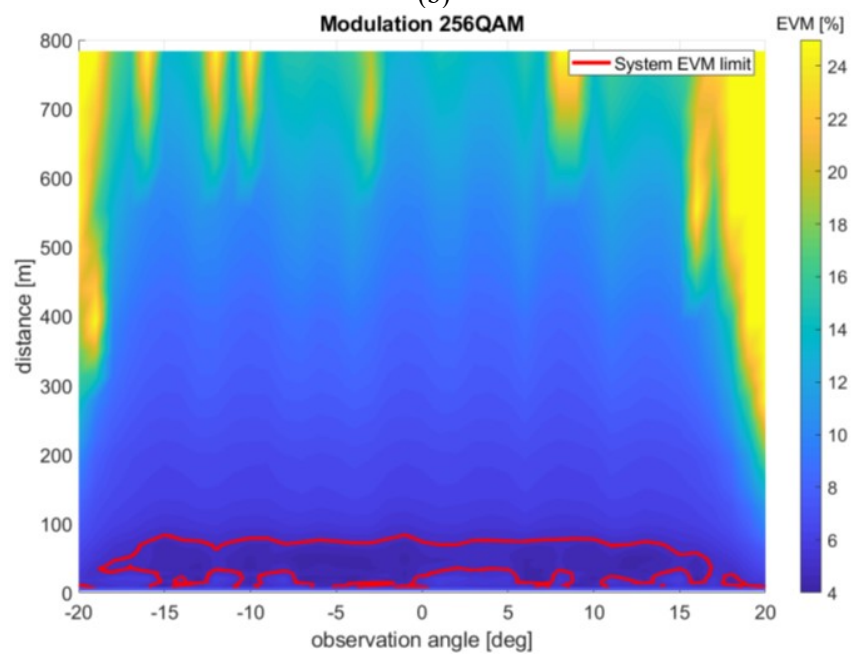

(c)

Fig. 21. Beam steering cell ranges for (a) 16-QAM, (b) 64-QAM and (c) 256-OAM based on EVM measurement results with $40 \mathrm{dBm}$ EIRP. between TX and RX EVMs. It was demonstrated that TX and RX AGCs in the common path of the RF phased array can be used to virtually extend the distance between TX and RX units by increasing signal attenuation between TX and RX. A good agreement of system EVM results measured in EMC chamber and 90-meter outdoor testing have been reported. The advantage of EMC chamber-based OTA system EVM performance measurements is that the link range estimations can be performed indoors without time consuming outdoor measurements.

It has been demonstrated that 256-QAM modulation can be used at $28 \mathrm{GHz}$ frequency for example as a mobile backhaul even though it has not been specified in 3GPP 5G NR specification for $\mathrm{mmW}$ operation. The OTA measurementbased link range estimation for maximum $5 \mathrm{G} \mathrm{mmW}$ link with $40 \mathrm{dBm}$ TX EIRP is $475 \mathrm{~m}$ in line-of-sight condition using 16QAM modulation, and $840 \mathrm{~m}$ with $45 \mathrm{dBm}$. The assumption is that TX and RX EVMs are equally contributing to the system EVM performance. The cell coverage estimation for 16-QAM modulation is $64800 \mathrm{~m}^{2}$. The link range is estimated for the 64-QAM as $366 \mathrm{~m}$ and $88 \mathrm{~m}$ for the 256-QAM modulation. The cell coverage areas are estimated and the coverage for the 64-QAM is $38950 \mathrm{~m}^{2}$ and for the 256-QAM is $2200 \mathrm{~m}^{2}$ with the equal system EVM partitioning between TX and RX EVMs. Measurements show that beam steering impacts RX EVM performance. At high RX input signal levels, EVM beam shape is different than with RX level close to the sensitivity level. Described measurement methods can be used to evaluate beam steering in a practical system.

The usable communication beamwidth of the $5 \mathrm{G}$ system changes based on used modulation order creating potential beam breathing phenomenon, since higher order modulations narrow usable communication beamwidth, and shorten the cell coverage causing potential problems in network planning.

\section{ACKNOWLEDGMENT}

The research leading to these results has received funding from the European Union H2020 5GPPP under grant n. 723247

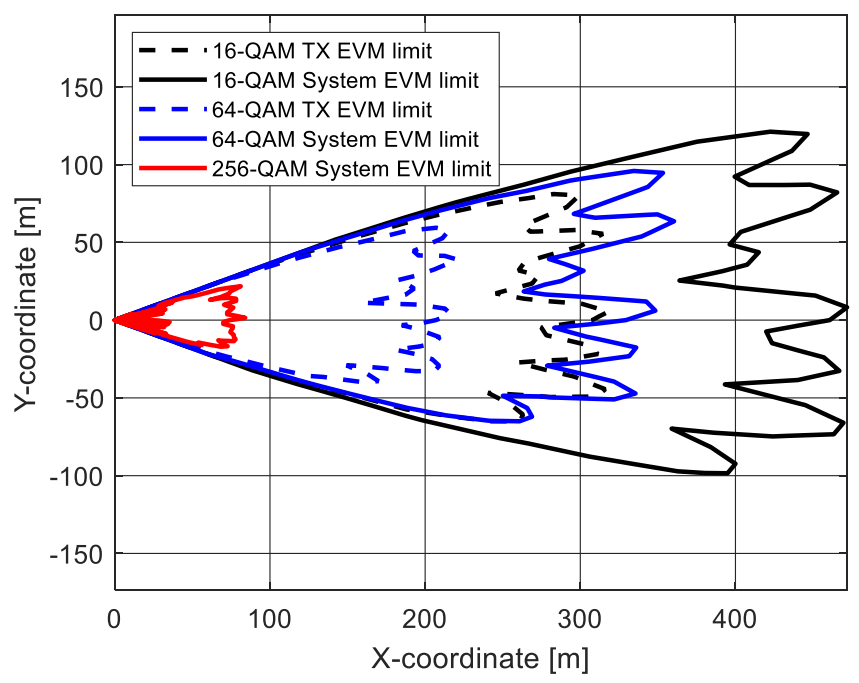

Fig. 22. Estimated cell coverages of $5 \mathrm{G}$ NR system based on measurements of $40 \mathrm{dBm}$ EIRP with different modulations and EVM limits based on table I. 
and supported by the Institute for Information \& communications Technology Promotion (IITP) grant funded by the Korea government (MSIP) (No.B0115-16-0001, 5GCHAMPION). Also, this work was supported by the Academy of Finland 6Genesis Flagship (grant no. 318927).

\section{REFERENCES}

[1] "AT\&T Completes World's First mmWave Mobile 5G Browsing Session with Standards-Based Mobile Device," AT\&T Inc., press release, Oct. 2018.

[2] "Customers in Chicago and Minneapolis are first in the world to get 5Genabled smartphones connected to a $5 \mathrm{G}$ network," Verizon Inc., press release, Apr. 2019.

[3] B. Sadhu et al., "A 28-GHz 32-Element TRX Phased-Array IC With Concurrent Dual-Polarized Operation and Orthogonal Phase and Gain Control for 5G Communications," in IEEE J. of Solid-State Circuits, vol. 52, no. 12, pp. 3373-3391, Dec. 2017.

[4] T. Sowlat et. al., "A 60GHz 144-Element Phased-Array Transceiver with $51 \mathrm{dBm}$ Maximum EIRP and $\pm 60^{\circ}$ Beam Steering for Backhaul Application," in ISSCC2018, 11. - 15. Feb. 2018, San Francisco, CA, pp. $66-68$.

[5] S. Shahramian et. al. "A Fully Integrated Scalable W-Band Phased-Array Module with Integrated Antennas, Self-Alignment and Self-Test," in ISSCC2018, 11. - 15. Feb. 2018, San Francisco, CA, pp. 74 - 76.

[6] Base Station (BS) radio transmission and reception (Release 15) 3GPP TS 38.104 V15.4.0, Dec. 2018.

[7] User Equipment (UE) radio transmission and reception, Part 2: Range 2 Standalone (Release 15) 3GPP TS 38.101 V15.2.0, Jun. 2018.

[8] Base Station (BS) radio transmission and reception (Release 15) 3GPP TS 36.104 V15.1.0, Dec. 2017.

[9] T. Tuovinen, N. Tervo and A. Pärssinen, "Analyzing 5G RF System Performance and Relation to Link Budget for Directive MIMO," IEEE Trans. Antennas Propag., vol. 65, no. 12, Dec. 2017, pp. 6636 - 6645.

[10] M. E. Leinonen, N. Tervo, M. Jokinen, O. Kursu, and A. Pärssinen, "5G mm-Wave Link Range Estimation Based on Over-the-Air Measured System EVM Performance," IMS2019, 2. - 7. Jun. 2019, Boston, MA

[11] S. Pellerano et al., "A Scalable 71-to-76 GHz 64-Element Phased Array Transceiver Module with $2 \times 2$ Direct Conversion IC in $22 \mathrm{~nm}$ FinFET CMOS Technology," ISSCC2019, San Francisco, CA, Feb. 17 -21, 2019, pp. $174-175$.

[12] J. Park, S. Lee, D. Lee and S. Hong, “A 28 GHz 20.3\%-Transmitter Efficiency 1.5०-Phase-Error Beamforming Front-End IC with Embedded Switches and Dual-Vector Variable-Gain Phase Shifters," ISSCC2019, San Francisco, CA, Feb. 17 -21, 2019, pp. 176 -177.

[13] O. Kursu et al., "Design and measurement of a $5 \mathrm{G} \mathrm{mmW}$ mobile backhaul transceiver at $28 \mathrm{GHz}$, EURASIP J. Wirel. Comm., Vol. 2018, No. 1, Aug. 2018.

[14] M. Sonkki et al., 'Linearly Polarized 64-element Antenna Array for mmWave Mobile Backhaul Application," in EUCAP Conf., London, UK, Apr. 9-13, 2018, pp. 1-5.

[15] M. Jokinen et. al., "Over-the-Air Phase Measurement and Calibration Method for 5G mmW Phased Array Radio Transceiver," ARFTG2019, 7. Jun. 2019, Boston, MA

[16] M. E. Leinonen, N. Tervo, O. Kursu and A. Pärssinen, "Out-of-Band Interference in $5 \mathrm{G} \mathrm{mmW}$ Multi-Antenna Transceivers: Co-Existence Scenarios," in European Conference on Networks and Communications, Ljubljana, Slovenia, 2018, pp. 1-5.

[17] J. J. Lee, "G/T and Noise Figure of Active Antennas," IEEE T. Antennas Propag., vol. 41, no. 2, Feb. 1993, pp. 241-244.

[18] M. E. Leinonen, G. Destino, O. Kursu, M. Sonkki and A. Pärssinen, " $28 \mathrm{GHz}$ Wireless Backhaul Transceiver Characterization and Radio Link Budget," ETRI J., Feb. 2018, 40: 89-100.

[19] M. Wiles and V. Rodriguez, "Choosing the Right Chamber for Your Test Requirements," Intern. J. of Electromagnetic Compatibility, May 2010.

[20] Base Station (BS) radio transmission and reception (Release 15) 3GPP TS 38.141 V15.1.0, Mar. 2019.

[21] H. K. Al-Musawi, W. P. Ng, Z. Ghassemlooy, C. Lu and N. Lalam, "Experimental analysis of EVM and BER for indoor radio-over-fibre networks using polymer optical fibre," in 20th European Conf. on Networks and Optical Comm., London, Jun. 30-Jul. 2, 2015, pp. 1-6.

[22] F. W. Breyfogle, Implementing Siz Sigma, Wiley \& Sons, USA, 1999.

[23] P. Susarla et al., "Smart-RF for mmWave MIMO Beamforming," in 29th PIMRC Conf., Bologna, Sept. 9 - Sept. 12, 2018, pp. 1-6.

[24] C. A. Balanis, Antenna Theory: Analysis and Design, $3^{\text {rd }}$ Ed., Wiley, 2005.

[25] M. D. Baba, M. Y. Ibrahim and F. J. A. Ghaffir, "Cell Breathing and Coverage Prediction in WCDMA," 2009 Intern. Conf. on Computer Technology and Development, Kota Kinabalu, 2009, pp. 287-290.

[26] N. Tervo et al., "Measurement Method for Characterizing Nonlinearity Under Near-Field and Far-Field Interferers in 5G mmW Phased Arrays," in EUMW2019, Paris, Sept. 29 - Oct. 4. 2019.

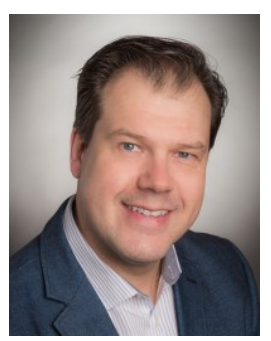

Marko E. Leinonen (S'17) Marko Leinonen received the M.Sc. and Licentiate in Technology degrees in electrical engineering from the University of Oulu, Finland, in 1996 and 2002. From 1994 to 2012 he was with Nokia Mobile Phones, Oulu, Finland working with various positions with radio engineering and technology management. From 2006 to 2007, he was a Senior Engineering Manager in Bangalore, India. From 2012 to 2016 he was a Master Developer with Ericsson, Oulu, Finland. Since 2017, he has been with University of Oulu, Centre for Wireless Communications, Oulu, Finland where he is currently a project manager. His research interests include wireless radio systems and quality topics in radio engineering. He holds more than 30 granted international patent families concentrating on radio engineering.

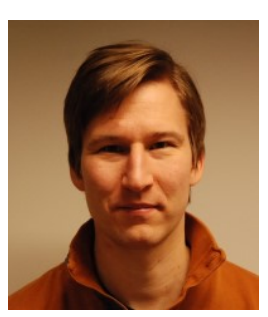

Markku Jokinen Markku Jokinen graduated as M.Sc. in Electronics from the University of Oulu in 2010 and he is working as a Research Scientist at Centre for Wireless Communications. $\mathrm{He}$ is pursuing the Doctoral degree and his has been recently focusing on the measurement techniques. He has gained experience on the design and implementation of wireless system algorithms and protocols with software-defined radio platforms.

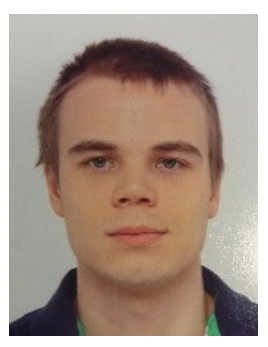

Nuutti Tervo (S'16) received the B.Sc. (Tech.) and M.Sc. (Tech.) degrees in electrical engineering from the University of Oulu, Oulu, Finland, in 2014. He joined the Centre for Wireless Communications (CWC), University of Oulu, in 2014 where he is currently working toward the Doctoral degree. He has a strong background in radio channel modelling, RF measurements and system level analysis. His current research interests include nonlinearity and linearization of millimeter-wave beamforming transceivers. 


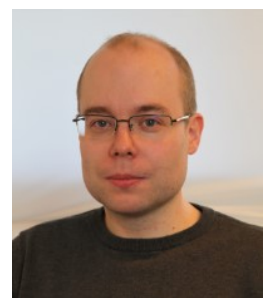

Olli Kursu Olli (Olli-Erkki) Kursu received his M.Sc. and D.Sc. degrees in electrical and electronics engineering from the University of Oulu, Oulu, Finland, in 2006 and 2015, respectively. Currently he is working as a postdoctoral researcher at the Centre for Wireless Communications in University of Oulu, Finland. His research interests include $\mathrm{mmW}, \mathrm{RF}$, analog and mixed signal circuit design for wireless communication systems.

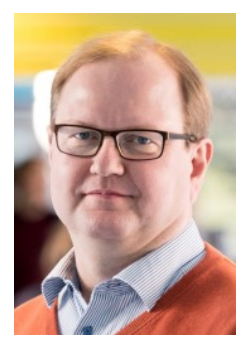

Aarno Pärssinen Aarno Pärssinen received the M.Sc., Licentiate in Technology and Doctor of Science degrees in electrical engineering from the Helsinki University of Technology, Finland, in 1995, 1997, and 2000, respectively. From 1994 to 2000 he was with Electronic Circuit Design Laboratory, Helsinki University of Technology, Finland, working on direct conversion receivers and subsampling mixers for wireless communications. From 2000 to 2011 he was with Nokia Research Center, Helsinki, Finland. From 2011 to 2013, he was at Renesas Mobile Corporation, Helsinki, Finland working as a Distinguished Researcher and RF Research Manager. From October 2013 to September 2014 he was an Associate Technical Director at Broadcom, Helsinki, Finland. Since September 2014 he has been with University of Oulu, Centre for Wireless Communications, Oulu, Finland where he is currently a Professor. His research interests include wireless systems and transceiver architectures for wireless communications with special emphasis on the RF and analog integrated circuit and system design. He has authored and coauthored one book, one chapter of a book, more than 50 international journal and conference papers and holds several patents. 